\title{
Responses of estuarine circulation to the morphological evolution in a
} convergent, microtidal estuary

\author{
Rui Zhang ${ }^{\mathrm{a}}$, Bo Hong ${ }^{\mathrm{b}}$, Lei Zhu ${ }^{\mathrm{a}, \mathrm{c}, \mathrm{d}}$, Wenping Gong ${ }^{\mathrm{a}, \mathrm{c}^{*}}$, Heng Zhang ${ }^{\mathrm{a}, \mathrm{c}, \mathrm{d}}$
}

a- School of Marine Sciences, SunYat-sen University, Guangzhou, China, 510275

b- School of Civil and Transportation Engineering, South China University of Technology, Wushan RD., Tianhe District, Guangzhou 510641, China

c- Southern Marine Science and Engineering Guangdong Laboratory (Zhuhai), Zhuhai

\begin{abstract}
d- Pearl River Estuary Marine Ecosystem Research Station, Ministry of Education,
\end{abstract}

$$
\text { Zhuhai, 519082, China }
$$

\section{Abstract:}

The Huangmaohai Estuary (HE) is a funnel-shaped microtidal estuary in the west of the Pearl River Delta (PRD) in southern China. Since China's reform and opening up in 1978, extensive human activities have occurred and greatly changed the estuary's topography, and modified its hydrodynamics. In this study, we examined the morphological evolution by analyzing remote sensing data with ArcGIS tools and studied the responses of hydrodynamics to the changes in topography from 1977 to 2010 by using the Delft3d model. We took the changes in estuarine circulation during neap tides in dry seasons as an example. The results show that human reclamation caused a narrowing of the estuary, and channel dredging deepened the estuary. These human activities changed both the longitudinal and lateral estuarine circulations. The longitudinal circulation was observed to increase with the deepening and narrowing of the estuary. The lateral circulation experienced changes in both the magnitude and pattern. The momentum balance analysis shows that when the depth and width changed simultaneously, the longitudinal estuarine circulation was modulated by both the * Supported by the National Natural Science Foundation of China under contract Nos 51761135021, 41506102 and 41890851.

** Corresponding author, E-mail:gongwp@mail. sysu. edu. cn 
channel deepening and width reduction, in which the friction, pressure gradient force, and advection terms were altered. The analysis of the longitudinal vortex dynamics indicates that the changes in the vertical shear of the longitudinal flow, lateral salinity gradient, and vertical mixing were responsible for the change in the lateral circulation. The changes in water depth are the dominant factor affecting lateral circulation intensity. This study has implications for sediment transport and morphological evolution in estuaries heavily impacted by human interventions.

Keywords: Estuarine circulation, Morphological evolution, Huangmaohai Estuary

\section{Introduction}

Estuarine circulation, the tidally averaged flow in estuaries including both the longitudinal and lateral circulations, is the main driving force for the transport of sediment, pollutants, and other materials, and also one of the primary factors affecting the ecological environment of estuaries (Kjerfve et al., 1981). Estuarine circulation is influenced by many factors (Geyer and Maccready, 2014), such as sea-level fluctuations (Wilson and Filadelfo, 1986), river discharge, tides (Pritchard, 1952), and winds (Scully et al., 2005; Waterhouse et al., 2013; Geyer and Maccready, 2014; Salles et al., 2015; Chen et al., 2020a). Topography in an estuary has a significant effect on the pattern and intensity of the estuarine circulation (Fischer, 1976; Dyer, 1977). Human activities may change the estuarine topography, leading to changes in the estuarine circulation and associated material transport. Therefore, a study of the estuarine circulation and its response to human activities is essential for integrated management of the development of estuarine resources, and the maintenance of the estuary's ecological health.

Channel deepening by dredging and sand mining is a common practice in the development and maintenance of navigable channels and resource utilization in estuaries. Generally speaking, channel deepening can increase the longitudinal 
estuarine circulation by decreasing the bottom friction and increasing the baroclinic forcing which is proportional to the water depth (Amin, 1983; Chernetsky et al., 2010; Winterwerp, 2011). On the other hand, the increase in water depth can also increase the salt intrusion and decrease the horizontal density gradient, thus reducing the baroclinic force. Channel deepening also affects estuarine circulation in other ways, such as increasing the Stokes transport and the associated compensating return flow (Amin, 1983), altering the nonlinear tidal rectification ( $\mathrm{Li}$ and O'Donnell, 1997), and tidal asymmetry in mixing between flood and ebb tides (tidal straining) (Simpson, 1990). Therefore, the effect of channel deepening is an intricate balance between these reinforcing and/or competing effects. Chant et al. (2018) demonstrated that a relatively small (15\%) increase in water depth can result in a double exchange flow. They attributed this increase to the increase in horizontal salinity gradient and/or a reduction in vertical mixing, but they did not give a clear distinction about how these two effects work together and which is dominant.

Change in estuary width is another aspect of topographic change in estuaries and is mainly caused by reclamation and utilization of salt marshes, construction of coastal protection structures along the estuarine banks. Change in estuary width generates a change in the estuarine convergence, and therefore a change in the estuarine circulation. Burchard et al. (2014) concluded that an increase in the estuarine convergence results in an enhancement or reduction of the longitudinal estuarine circulation as increased estuarine convergence can reduce or even reverse the straining-induced circulation, though the advection-induced circulation is increased. Changes in estuarine width can also modify the lateral circulation and feedback to the generation of the longitudinal estuarine circulation through the change in lateral advection (Lacy et al., 2003; Lerczak and Rockwell Geyer, 2004; Scully et al., 2009; Burchard et al., 2010; Burchard et al., 2014). Lerczak and Rockwell Geyer (2004) suggested that lateral effects on the longitudinal estuarine circulation would be stronger in narrower estuaries given a constant lateral salinity gradient. Schulz et al. (2015) investigated the impact of the depth-to-width ratio of the estuarine cross-section on the longitudinal estuarine 

maximum in medium-wide channels. They diagnosed the mechanisms for such a phenomenon and attributed it to the sensitivities of the straining- and advection-induced circulations on the changes in depth-to-width ratio.

As revealed by Lerczak and Geyer (2004) and other researchers (Chen et al., 2020b), lateral processes play important roles in the generation of the longitudinal

91 estuarine circulation. The lateral circulation can modify the longitudinal estuarine

92 circulation by the lateral redistribution of the longitudinal current in a direct way

93 (Lerczak and Geyer, 2004), and change the longitudinal pressure gradient force and the

94 friction in an indirect way. In estuaries, the pattern and intensity of lateral circulation are controlled by three processes ( $\mathrm{Li}$ et al., 2014): vertical shear of the longitudinal current affecting the tilting of planetary vorticity, lateral salinity gradient (baroclinicity), and diffusion. The longitudinal estuarine circulation can affect the lateral circulation through all the mentioned three factors. Therefore, the interaction between the longitudinal and lateral processes is fully nonlinear and quite complex. Though these interactions have been discussed in detail (Scully et al., 2009; Li et al., 2017), several questions remain open: How does the longitudinal estuarine circulation affect the intensity and vortex structure of the lateral circulation? Does a decreased/increased lateral circulation necessarily lead to a weakened/strengthened longitudinal circulation? These questions become complicated in an estuary where both width and depth vary. Previous studies showed that the narrowing and deepening of the Yangtze River Estuary resulted in an enhanced longitudinal estuarine circulation (Zhu, 2018), which changed from transversely sheared to vertically sheared. The estuarine stratification was also found to be strengthened, along with an increase in the intensity of lateral circulation. Zhu et al. (2015) investigated the influences of channel deepening and widening on the tidal and nontidal circulations of Tampa Bay, USA, and found that the nontidal circulation was strengthened by these human interventions. However, how does the estuarine circulation respond to both narrowing and deepening/shallowing of the estuary? What happens when the narrowing rate is much larger or smaller than the 

cross-section widths between two consecutive years divided by the width in the earlier year. Similarly, the deepening rate is the ratio of the difference of water depth in the cross-section between the two consecutive years divided by the earlier year's depth.

Here we try to address the above questions by studying the changes in the estuarine circulation via using the historical topographic data obtained from 1977 to 2010 in the Huangmaohai Estuary (HE). The HE is a microtidal estuary in the southwest of the Pearl River Delta (PRD), and experienced different stages of topographic changes under human activities from 1977 to 2010, which can be grouped into two scenarios: narrowing and deepening (1977-1994, and 2003-2010), and narrowing and shallowing (1994-2003). These two scenarios of topographic changes caused different responses of the estuarine circulation, and thus provided a good opportunity to study the effect of human activities induced morphological evolution on the estuarine circulation.

In this study, we used a state-of-the-art three-dimensional baroclinic model (Delft 3d) to simulate the changes in hydrodynamics in the HE in different years and examined the changes in intensities of the longitudinal and lateral estuarine circulations, followed by an analysis of the mechanisms for these changes by conducting diagnostic analyses of the momentum balance. The structure of the rest of the paper is as follows. Section 2 introduces the study area and numeral model (including model setting and validation). Section 3 presents the results of morphological evolution and changes in the estuarine circulation. Then, the mechanisms for the changes in estuarine circulation are investigated using the momentum and vortex balance equations in Section 4. Finally, the conclusions are presented in Section 5. For the sake of conciseness, we append a supplement in the appendix about the model validation for water level, current, and salinity.

\subsection{Study area}


The HE is located in the west of the PRD in southern China and exhibits a distinctly convergent geometry, with a latitude ranging from $21^{\circ} 50^{\prime}$ to $22^{\circ} 13^{\prime} \mathrm{N}$ and a longitude ranging from $113^{\circ} 00^{\prime}$ to $113^{\circ} 51^{\prime} \mathrm{E}$ (Fig. 1). The estuary is composed of a bay (Huangmao Bay) and a tidal river. The bay is trumpet-shaped with an area of 409 $\mathrm{km}^{2}$. It has a complex bathymetry comprising of two channels and three shoals, namely the West Channel and East Channel, the West Shoal, Middle Shoal, and East Shoal. In recent decades, the West Channel is observed to shrink and almost disappear now (Jia et al., 2012). The width of the bay is $30 \mathrm{~km}$ at the estuary mouth and decreases to 1.8 $\mathrm{km}$ at the head. The mean water depth of the bay is $4.5 \mathrm{~m}$ (Gong et al., 2014). The bay is connected to the upstream river catchment by two constrictions (Yamen and Hutiaomen Outlets). Several islands, namely Dajin Island, Hebao Island, and Gaolan Island, are scattered at the estuary's mouth. The estuary mouth is divided into three entrances: the West Entrance between the Dajin Island and the western shore; the Central Entrance between the Dajin Island and the Hebao Island, where the West Channel passes through; and the East Entrance between the Hebao Island and the

Gaolan Island, where the East Channel goes through (shown in Fig. 1b).

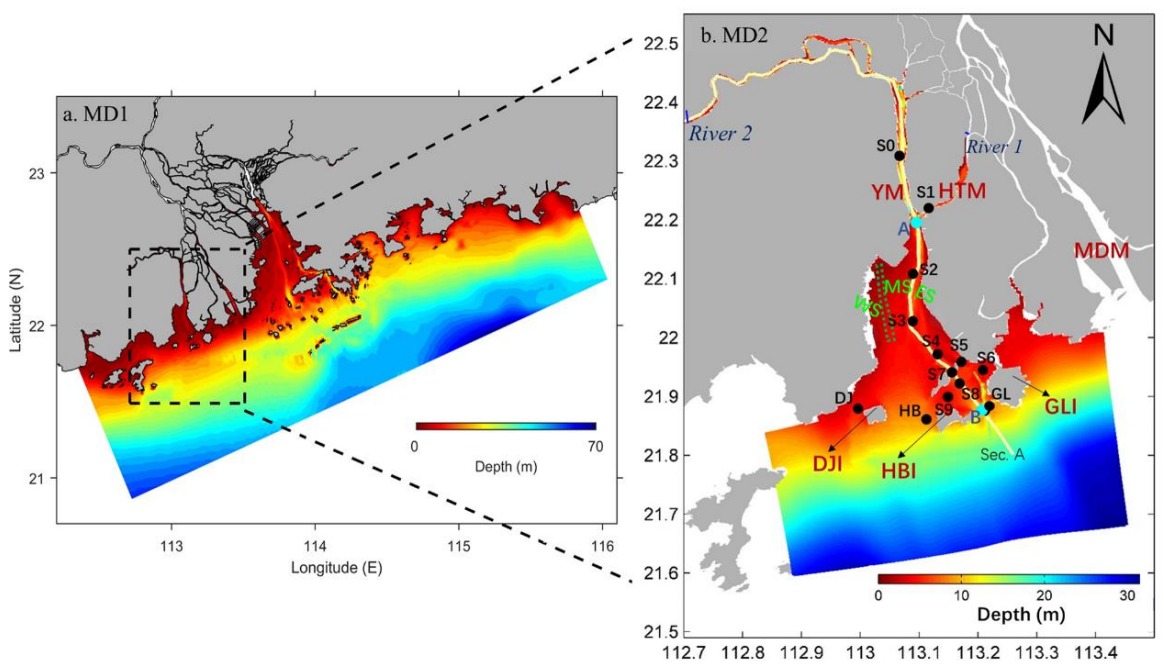


Fig. 1. The study area (Huangmaohai estuary) and observation stations. Major topographic features and domains of the nested modeling system over (a) the PRD and (b) the HE and its adjacent waters. $\mathrm{YM}=$ Yamen; HTM = Hutiaomen; $\mathrm{MDM}=$ Modaomen; DJI = Dajing Island; GLI = Gaolan Island; HBI = Hebao Island. The black dots (S0-S9, DJ, HB, and GL) in the MD2 domain are stations of field deployments in March 2010. The solid lines represent the along-channel transect (Section A (AB)), which lies in the East Channel. The green dotted lines represent the West Channel in 1977. Three shoals in (b): West Shoal (WS), Middle Shoal (MS), and East Shoal (ES).

The HE has a subtropical monsoon climate, with the precipitation in the wet season (from May to September) being high. Approximately $80 \%$ of the river discharge occurs during the wet season, with an average discharge of $200.23 \mathrm{~m}^{3} / \mathrm{s}$. The tides in the HE are mixed semidiurnal with dominant semi-diurnal constituents and smaller diurnal constituents. The tides show obvious asymmetry in terms of tidal duration, velocity, and current acceleration between floods and ebbs (Gong et al., 2016). The tidal range is approximately $1.5 \mathrm{~m}$ at the mouth and experiences an initial increase from the mouth towards the head owing to a strong convergence of the bay width. Further landward in the tidal river beyond the bay head, the tidal range decreases by the overwhelming bottom friction (Gong et al., 2012). The tidal current velocity ranges from $0.5 \mathrm{~m} / \mathrm{s}$ to $1.5 \mathrm{~m} / \mathrm{s}$ (Huang, 2011), and is higher in deep channels than on shallow shoals. The tidal currents are generally rectilinear in deep channels but become more rotary in shallow shoals.

Since the 1980s, human activities have been intense in the HE estuary. A hydroelectric power project upstream of the estuary, channel dredging, sand mining, and construction of Gaolan Island levees have led to great changes in the HE's topography. Also, the HE has rich tidal flat resources and endured frequent reclamation activities. From 1965 to 2003 , a total of $142.29 \mathrm{~km}^{2}$ tidal flat was reclaimed, with an average reclamation rate of $3.74 \mathrm{~km}^{2} / \mathrm{a}$, and the reclamation rate continuously but gradually increased during that period. After 2003, the reclamation rate slowed down. In terms of channel dredging, the Yamen Waterway Project was conducted in 1997 to deepen the channel between S0 and S3 in Fig. 1b (Luo, 2010). In April 2005, the Yamen 
193 Channel regulation project was implemented to alleviate the serious siltation in the channel, with the channel being dredged to a depth of about $6 \mathrm{~m}$.

In the following, we chose 1977, 1994, 2003, and 2010 as the representative years to study the typical scenarios of bathymetric changes in the HE.

\subsection{Remote sensing and topographic data}

199
Remote sensing data were used for coastline extraction and included Landsat Multi-Spectral Scanner (MSS) data, Landsat Thematic Mapper (TM) data, and Landsat Operational Land Imager (OLI) data. A total of 142G data of 66 images (Table 1) covering the PRD during cloudless days in multiple years (from 1973 to 2018) were downloaded from http://www.gscloud.cn/. These data were firstly processed by geometric and atmospheric corrections by the ENVI 5.3 software. Subsequently, they were compared with topographic data for further geometric corrections. The errors were shown to be less than 0.5 pixels (Ai et al., 2019). The topography data inside the HE were derived from nautical charts (1977, 1994, 2003, and 2010), published by the Navigation Safety Guarantee Bureau. The filling and excavation toolbox of ArcGIS was used to calculate the difference between the volumes in two consecutive periods by superimposing the corresponding Digital Elevation Models (DEM). This method is actually to decompose three-dimensional space entities into many cuboids, then calculate the differences of area and volume of each cuboid between two consecutive years, and classify the cuboids into different categories based on the siltation thickness. We thus obtained the average siltation rates of the study area over different years (Figs. $2 \mathrm{a}-\mathrm{c})$. 

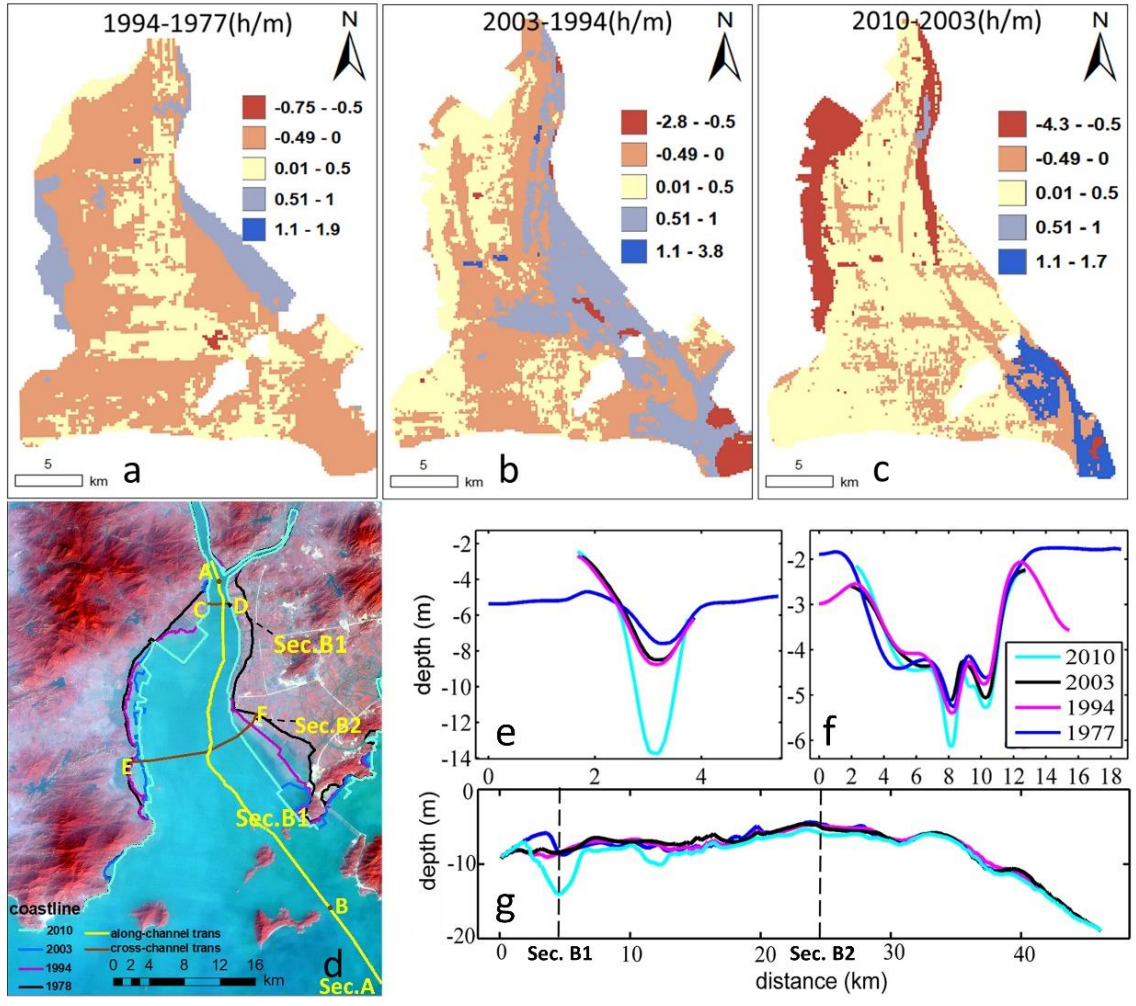

Fig. 2. (a-c) Water depth difference between two consecutive years ((a)1994-1977; (b)20031994; (c)2010-2003), where the positive value indicates "deepening" and the negative one indicates "siltation", (d) Shorelines of 1977-2010 and locations of two cross-sections (AB: Sec. A; CD: Sec. B1; EF: Sec. B2); (e, f, and g) The bathymetric evolutions at Sections B1, B2, and A in 1977, 1994, 2003, and 2010.

Table 1. Data of remote sensing images

\begin{tabular}{llllll}
\hline Time & Satellite & Image sensor & Resolution/m & Path/Row & $\begin{array}{l}\text { Memory } \\
\text { space }\end{array}$ \\
\hline 1973,1978 & Landsat3 & MSS & 78 & & \\
$1986-2011$ & Landsat5 & TM & 30 & $122 / 45$ & $142 \mathrm{G}$ \\
2012 & Landsat7 & ETM & 30 & & \\
$2013-2018$ & Landsat8 & OLR & 30 & & \\
\hline
\end{tabular}


The numerical model Delft3d was used to simulate the hydrodynamics in the HE. The Delft3d model is a fully three-dimensional hydrodynamic water quality model, and can accurately simulate large-scale flow, water quality, and morphological evolution (Lesser et al., 2004). The algorithm of the Delft $3 \mathrm{~d}$ model can guarantee the conservation of mass, momentum, and energy. The model grid consisted of a nesting grid system, with the MD1 (parent model, Fig. 1a) covering the whole PRD, and the MD2 (child model) covering the HE. For the MD2 model, an orthogonal Cartesian horizontal grid of $269 * 620$ was established, with the horizontal resolution ranging from $85 \mathrm{~m}$ in the channel to $324 \mathrm{~m}$ at the ocean boundary. Vertically, the grid was discretized into 10 layers of $\sigma$ coordinate. The model system used here is the same as the one in Chen et al. (2020a). Briefly, the open boundary conditions of the MD1 model included atmospheric forcing at the water surface, river discharge at the upstream boundary, tidal and non-tidal water elevations and currents, a constant salinity of 34 psu at the open ocean boundary. The results from the MD1 were interpolated to provide ocean boundary conditions for the MD2 model.

As mentioned above, the hydrodynamics in the HE experiences distinct seasonal variation. The estuarine circulation during the wet season has been extensively studied before (Chen et al., 2020a; Chen et al., 2020b). Here we choose the dry season to investigate the changes in the estuarine circulation caused by topographic changes in different years. We conducted a series of numerical experiments using the bathymetry data in $1977,1994,2003$, and 2010. The simulation time was chosen to be from 00:00 on March 1 to 23:00 on March 31 in the dry season, when observation data were available in 2010. Field measurements were carried out at 14 mooring stations on March 17th 17:00 to 18th 22:00, 2010. In these surveys, ADCP was utilized to measure the vertical profile of current with a vertical resolution of $0.3 \mathrm{~m}$ and a time resolution of $5 \mathrm{~min}$, and CTD was used to measure the vertical profiles of salinity and temperature 
259 for model validation here. In all the four scenarios, two upstream boundaries were specified (Fig. 1b): at River 2 by specifying real-time water level data from the MD1 model from 00:00 on March 1, 2010, to 23:00 on March 31, 2010, with a time interval of 1 hour; At River 1 by specifying a constant river discharge of $100 \mathrm{~ms}^{-2}$. The choice of this constant value was based on the previous simulation experiences (Chen et al., 2020a; Chen et al., 2020b). The salinities at the river inflow boundaries were set to be 0 psu. The only changing condition of the four scenarios was the topography (Table 2), so the effect of topographic change can be identified. The measured data from 14 stations in 2010 were used to validate the model. The validation included water level, current direction and magnitude, and salinity (Figs. A. 1-4).

Table 2. Coastline, bathymetries, salinity, flow, and tidal boundary in the four model scenarios.

\begin{tabular}{llllll}
\hline Scenario & Coastline & $\begin{array}{l}\text { Bathymetrie } \\
\text { s }\end{array}$ & $\begin{array}{l}\text { The salinity } \\
\text { of the open } \\
\text { sea }\end{array}$ & $\begin{array}{l}\text { Tidal } \\
\text { boundary }\end{array}$ \\
\hline $1977 / 03$ & 1977 & 1977 & $2010 / 03$ & $2010 / 03$ & $2010 / 03$ \\
$1994 / 03$ & 1994 & 1994 & $2010 / 03$ & $2010 / 03$ & $2010 / 03$ \\
$2003 / 03$ & 2003 & 2003 & $2010 / 03$ & $2010 / 03$ & $2010 / 03$ \\
$2010 / 03$ & 2010 & 2010 & $2010 / 03$ & $2010 / 03$ & $2010 / 03$ \\
\hline
\end{tabular}

In this study, the Willmott skill score (SK) was used to evaluate whether the model result is consistent with the observed data (Willmott, 1981). The SK is defined as:

$$
S K=1-\frac{\sum_{i=1}^{n}\left(O_{i}-M_{i}\right)^{2}}{\sum_{i=1}^{n}\left[\left|M_{i}-\bar{O}\right|+\left|O_{i}-\bar{O}\right|\right]^{2}},
$$

where $n$ is the number of the observed data, $M$ and $O$ are model simulation results and observations, respectively, and $\bar{o}$ is the average value of the observation data. SK is used to measure the consistency between the model results and the observations, with a value between 0 and 1 . The larger the value is, the more consistent the simulation results are with the observed data.

Firstly, the water level of the MD2 model was validated (Fig. A. 1). The SKs of the four observed stations are all above 0.86 , indicating that the water level simulation is reasonable. Secondly, the modeled current direction showed good performance except 
283 for the surface layer at Stations DJ and S0 (Fig. A. 2), almost all the SKs are greater 284 than 0.7 (Table 3). The simulation of current speed (Fig. A. 3) is worse than that of the 285 current direction, but the SKs at most stations are above 0.6, showing a good 286 performance. We note that the SK (less than 0.4) at Station S3 is significantly lower 287 than other stations, mostly because the station was located near the null point (the 288 convergence point between seaward and landward bottom residual flows) and the 289 current speed was quite small. Lastly, the trends of observed and simulated salinities 290 are consistent (Fig. A. 4), and almost all the SKs of salinity validation are above 0.5, 291 especially in S1-S3, showing a good performance of the salinity simulation.

Table 3. Skill scores by comparison of modeled results with observations.

\begin{tabular}{llllllllll}
\hline \multirow{2}{*}{ Stations } & \multicolumn{3}{l}{ Current direction } & \multicolumn{3}{c}{ Current speed } & \multicolumn{3}{c}{ Salinity } \\
& Sur & Mid & Bot & Sur & Mid & Bot & Sur & Mid & Bot \\
\hline S0 & 0.18 & 0.96 & 0.96 & 0.77 & 0.88 & 0.86 & 0.32 & 0.35 & 0.35 \\
S1 & 0.94 & 0.99 & 0.99 & 0.65 & 0.66 & 0.61 & 0.94 & 0.94 & 0.90 \\
S2 & 0.78 & 0.79 & 0.71 & 0.83 & 0.84 & 0.84 & 0.84 & 0.85 & 0.85 \\
S3 & 0.87 & 0.98 & 0.95 & 0.34 & 0.38 & 0.39 & 0.92 & 0.79 & 0.77 \\
S4 & 0.84 & 0.94 & 0.94 & 0.53 & 0.55 & 0.53 & 0.77 & 0.64 & 0.54 \\
S5 & 0.86 & 0.92 & 0.93 & 0.66 & 0.71 & 0.72 & 0.37 & 0.25 & 0.26 \\
S6 & 0.79 & 0.90 & 0.88 & 0.68 & 0.75 & 0.74 & 0.15 & 0.20 & 0.25 \\
S7 & 0.82 & 0.85 & 0.96 & 0.74 & 0.79 & 0.83 & 0.86 & 0.66 & 0.56 \\
S8 & 0.84 & 0.89 & 0.89 & 0.59 & 0.62 & 0.66 & 0.82 & 0.77 & 0.72 \\
S9 & 0.80 & 0.74 & 0.77 & 0.54 & 0.46 & 0.41 & 0.59 & 0.50 & 0.52 \\
DJ & 0.61 & 0.77 & 0.77 & 0.38 & 0.47 & 0.51 & 0.66 & 0.47 & 0.37 \\
GL & 0.89 & 0.91 & 0.93 & 0.50 & 0.51 & 0.49 & 0.37 & 0.43 & 0.41 \\
HB & 0.71 & 0.89 & 0.89 & 0.60 & 0.56 & 0.56 & 0.57 & 0.54 & 0.53 \\
\hline
\end{tabular}

As a whole, the simulation of surface currents is worse than that in other layers, since winds and waves were not included in our model simulations, in which the surface flow is more susceptible to these forcings. The specified river flow at River 2 was constant, which may deviate from the real-time data (not available), leading to a poor salinity reproduction at upstream stations. In short, the water level and current are wellvalidated. The simulation of salinity is generally good, except for some deviations at upstream stations. It shows that the model can reasonably simulate the hydrodynamic 


\section{Results}

\subsection{Morphological evolution}

Morphological changes between 1977, 1994, 2003, and 2010 are shown in Figs. 2a-c. According to the pattern of erosion and siltation in the two consecutive years, most areas in the HE experienced siltation from 1977 to 1994. The East Channel was deepened by about $0-0.5 \mathrm{~m}$. In the middle of the bay, the nearshore areas were under erosion, and the erosion thickness at the eastern shore was twice that at the western shore. In other areas, the siltation thickness was between 0 and $0.5 \mathrm{~m}$. From 1994 to 2003, erosion occurred in the West Shoal, East Channel, East Shoal, and Middle Shoal. Siltation of 0.01-0.5 m happened in the rest of the area, which accounted for most of the HE, so the HE became shallower in 2003. From 2003 to 2010, the West Shoal became significantly shallower with a siltation thickness of about $0.5-1 \mathrm{~m}$. The East Shoal almost disappeared, and its relict area endured siltation of 1.1-1.7 m, which was mainly due to the construction of coastal protection works. Strong erosion occurred in other areas, especially in the upper bay with a deepening of more than $4 \mathrm{~m}$, and the overall water depth of the HE became greater in 2010. East Channel experienced continuous erosion before 1977. From 1977 to 2003, the channel was under siltation, particularly at the river mouth bar (between the upper bay and the entrances, see Gong et al. (2014), whereas at the upper bay (from the head to the null point of the East Channel, here the null point means the convergence point at which the upstream seaward flow meets the downstream landward bottom flow) and at the inter-island sections, the channel was under erosion. In 2003, siltation in the East 

in 2006 (Luo, 2010).

Overall, the water depth of the HE changed considerably from 1977 to 2010. It first experienced erosion, then underwent siltation, and followed by erosion again.

Figure $2 \mathrm{~d}$ shows the changes of coastlines for the four representative years. To calculate the rate of geometry convergence, the DSAS tool (Version 5.0) in Arcmap 10.3 was used to calculate the end-point rates for cross-shore transects. A more detailed procedure is in Zhang et al. (2019). We chose one longitudinal section along the channel in the estuary and two cross-sections (in Fig. 2d) along the channel for analysis. The longitudinal section (Sec. A) extends from the bay head (point A in Fig. 1b) to the estuary mouth (point B in Fig. 1b), spanning a distance of $50 \mathrm{~km}$. Sec. B1 is located at about $4 \mathrm{~km}$ downstream from the bayhead, where the water depth changes sharply in the lateral (or longitudinal) direction (see Fig. 2e). Sec. B2 is approximately $24 \mathrm{~km}$ downstream from the bayhead and near the null point in the middle of the estuary (see Fig. 2f), and the width of the estuary varied dramatically here (see Fig. 2e). At Sec. A, the water depth near the point of Sec. B1 endured a great change in 2010 due to channel dredging (Fig. 2g). In other periods, the water depth along its course endured gradual deepening. At Sec. B1, the bathymetric change is featured by an increase in water depth and negligible change in width over time. At Sec. B2, both the water depth and width experienced changes from 1977 to 2010, with the depth increased and width decreased (Fig. 2f). The above three sections clearly depict the topographic changes of the estuary in different years.

\section{2 changes in the vertically averaged flow and salinity}

Here we present the changes in the tidally and vertically averaged flow and salinity during neap tides in different years in Fig. 3. In 1977 (Fig. 3a), the current speed was generally small, except at the inter-island sections and in the channel. The vertically averaged flow was seaward in the upper bay and the right part of the lower bay (looking 

speed was increased in the channel, particularly near Sec.B1. The overall flow pattern was almost similar to that in 1977. The salt intrusion was increased, as the $10 \mathrm{psu}$ isohaline intruded to a latitude of $22.15^{\circ} \mathrm{N}$. In 2003 (Fig. 3c), the flow pattern still kept unchanged when compared to that in previous periods. The current speed was decreased relative to that in 1994. However, the salt intrusion became aggravated as the $10 \mathrm{psu}$ isohaline had reached the bayhead at the Yamen Outlet. In 2010 (Fig. 3d), the seaward flow became more dominant in the upper bay, and more biased southwestward. The seaward flow in the channel was greater than in 2003. The salt intrusion was more serious than in 2003, as the 10 psu isohaline reached beyond the bayhead and entered into the tidal river of the estuary.

Overall, we observed that the tidally and vertically averaged flow during neap tides experienced an increase-decrease-increase by the topographic changes, whereas the saltwater consistently intruded further into the estuary.

As a supplement, we present the horizontal distributions of tidally averaged surface and bottom circulation and salinity during neap tides for different years in the appendix (Figs. A. 5 and 6). Over the study period, the enhancement of salt intrusion

377 was stronger for the bottom layer and weaker for the surface layer, whereas the increase

378 in residual flow was stronger in the surface layer and weaker in the bottom layer. 


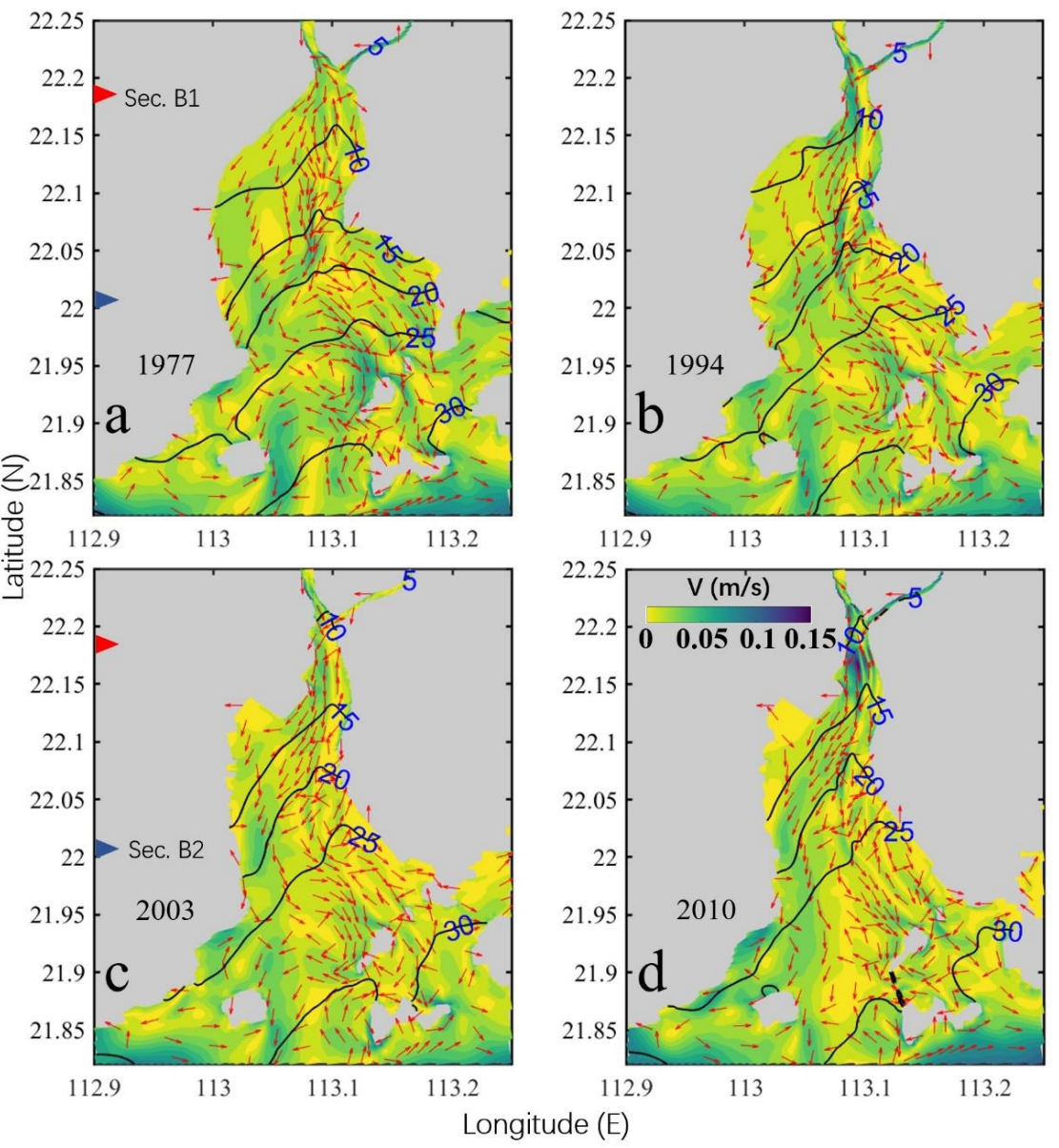

Fig. 3. Patterns of the vertical-averaged horizontal circulation during neap tide in 1977(a1), 1994(a2), 2003(a3), and 2004(a4). The magnitude of the current is represented by the color shading, while the current direction is shown by the arrows. The salinity is depicted by the contour lines. The red and blue triangles depict the positions of two cross-sections (Sec.B1 and Sec.B2).

\subsection{Changes in the estuarine circulation}

Figures 4 a-d present the longitudinal estuarine circulation and the distributions of salinity isolines during the neap tide in the longitudinal section (Sec. A in Fig. 1b) in different years. The results show that the upper part of the estuary (upstream of the null 
391 point) was highly stratified, and the lower part of the estuary (downstream of the null

392 point) was well mixed. The classical exchange flow structure of "a landward residual

393 flow near the bottom and a seaward residual flow near the surface" was more distinct

394 upstream of the null point. Over time, the surface seaward flow became stronger and

395 more concentrated with the narrowing of the estuary, particularly in 2010. It extended

396 more downstream to near the estuary mouth with the narrowing of the estuary, as

397 evidenced by the extent of the seaward flow of $0.2 \mathrm{~m} / \mathrm{s}$. Concomitantly, the bottom

398 landward flow was strengthened and concentrated with the increase in depth.

399 We also present the changes in the surface and bottom current horizontally. Figs. 4e1-g1 show that when the estuary deepened (1977-1994 and 2003-2010), the surface current speed increased in the channel, and when the estuary shoaled (1994-2003), the surface current in the channel decreased. The changes in the bottom current showed a similar trend (Figs. 4e2-g2), except at the upper part of the channel from 1977 to 1994 , in which the width was considerably narrowed.

Along with the change in the longitudinal estuarine circulation, the salt intrusion at Sec. A did not change significantly from 1977 to 1994, but increased from 2003 on, particularly in 2010, when the isohaline of 15 psu reached Sec.B1, whose salinities were less than $12 \mathrm{psu}$ in previous years (Figs. 4a-d). The salt intrusions at the surface and bottom gradually increased with the estuary narrowing (Figs. 4e1-g2). 


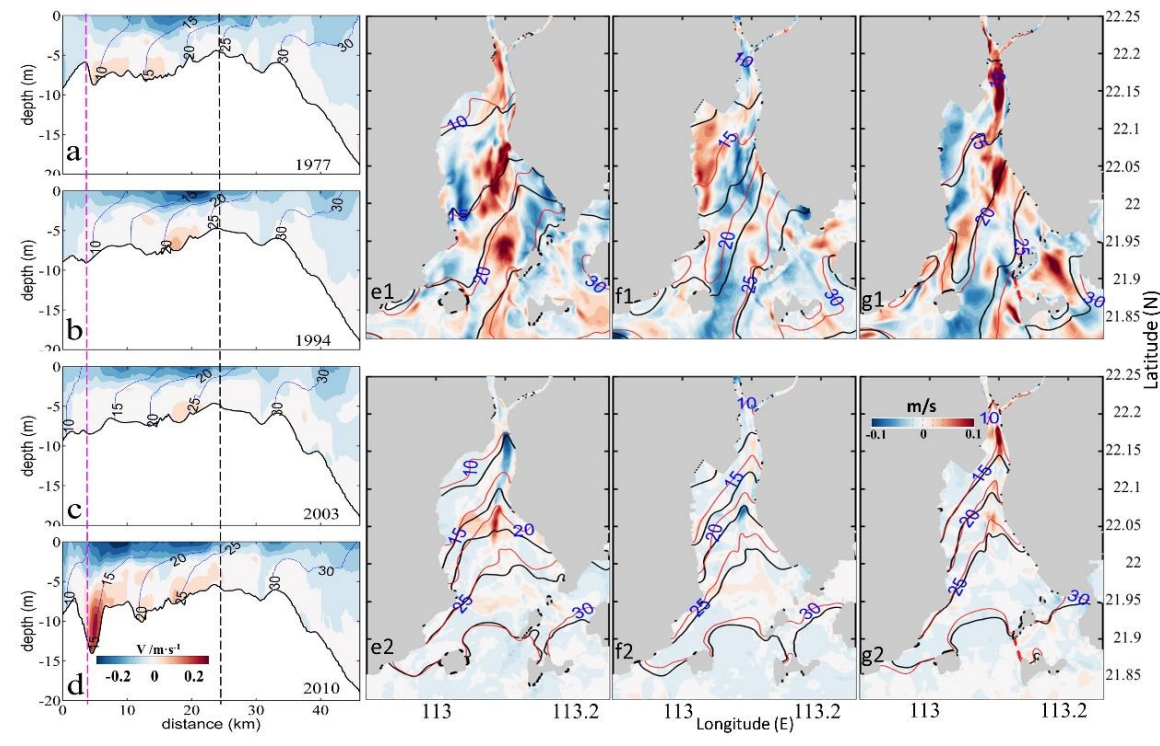

Fig. 4. The patterns of the estuarine circulation during the neap tide in March 1977(a), 1994(b), 2003(c), and 2010(d). The thin lines are the isolines of salinity in a-d. The pink and black dotted lines represent the locations of Secs. B1 and B2, respectively. The starting point of the X-axis is Point A in Fig. 1b. Surface current differences from 1977 to 1994(e1), from 1994 to 2003(f1), and from 2003 to 2010(g1); Bottom current differences from 1977 to 1994(e2), from 1994 to 2003(f2), and from 2003 to 2010(g2). The red and black lines represent the isolines of salinity in the later year and the earlier year.

To analyze the changes of lateral circulation in the estuary, we show the structure and intensity of the lateral circulation at the two cross-sections (Figs. 5 and 6).

At Sec. B1 (Fig. 5), with the increase of water depth, the salinity difference between the surface and bottom increased, along with an increase in the bottom salinity, which is more than 14 psu in 2010. For the lateral circulation, there was no distinct gyre structure in 1977 , but a pair of opposite vortices started to develop at the position of $3.5 \mathrm{~km}$. In 1994, the lateral flow was dominated by an eastward flow. In 2003, a clockwise vortex was developed over the West Shoal (0.5-1 km). Meanwhile, an anticlockwise circulation with smaller vortex intensity was developed in the region of $1-2 \mathrm{~km}$. Another clockwise circulation was developed over the East Shoal. When the 

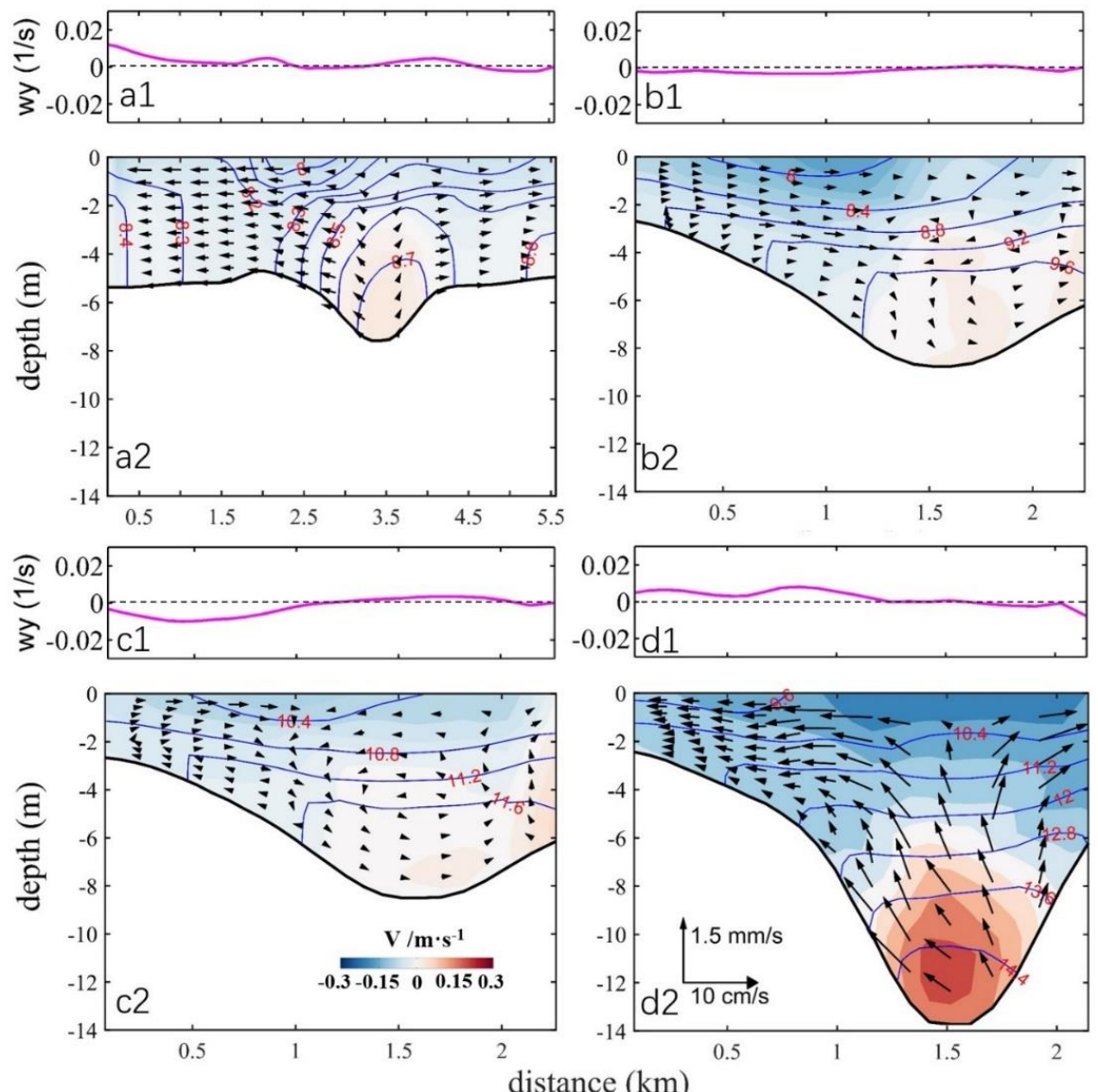

Fig. 5. The lateral circulation and isohalines (blue lines) at Sec. B1 in 1977(a2), 1994(b2), 2003(c2), and 2010(d2). The starting point of the $\mathrm{X}$-axis is Point $\mathrm{C}$ in Fig. $2 \mathrm{~d} . w_{y}$ is the longitudinal vorticity at Sec. B1 in 1977(a1), 1994(a2), 2003(a3), and 2010(a4).

Figure 6 shows the changes in lateral circulation at Sec. B2. With the decrease of estuary width, the salinity increased in the cross-section over the years. There developed a clockwise circulation at the right of the deep channel in 1977 and 1994. This clockwise vortex was seen to move westward from 2003 on. The spatial extent of the clockwise circulation in the deep channel increased significantly over time. Clockwise vortices developed over the East Shore from 1977 to 2010, but their intensity 
442 became weaker since 2003. In 1977 and 1994, the distance between the deep channel 443 and the East Shore was greater than $2 \mathrm{~km}$, and the accommodation space was enough 444 for the clockwise vortices to develop sufficiently. From 2003 on, the accommodation 445 space at the East Shore became limited and restricted the full development of the 446 clockwise vortex. Over the West Shoal, the lateral circulation pattern showed an 447 anticlockwise circulation in 1977 and 1994. However, since 2003, the lateral circulation 448 over the West Shoal began to develop a two-cell pattern, with an anticlockwise gyre at 449 the surface and a clockwise one near the bottom. The clockwise cell developed well in 450 2010.
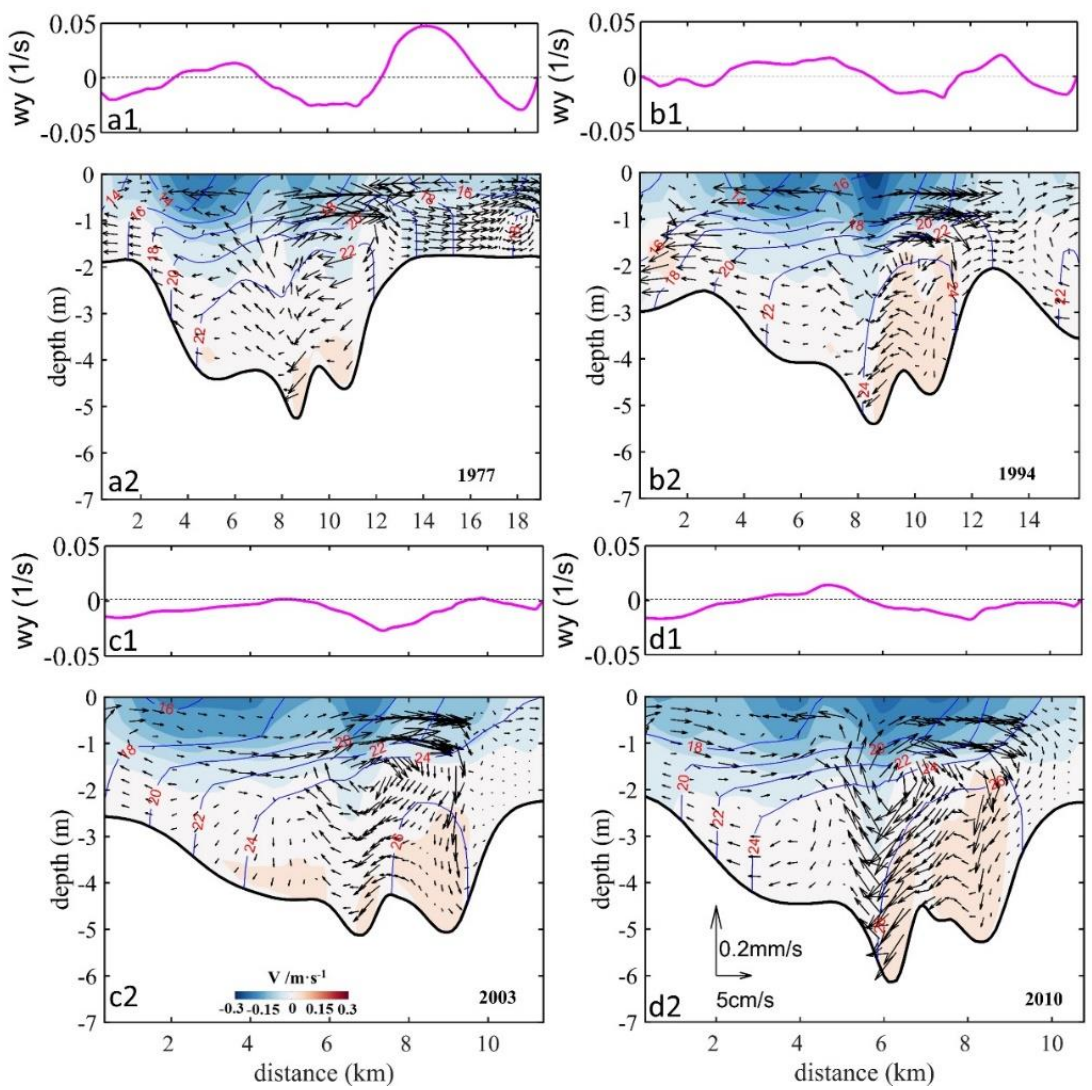

Fig. 6. The distribution of lateral circulation and isohalines (blue lines) at Sec.B2 in 1977(a2), 1994(b2), 2003(c2), and 2010(d2). The starting point of the X-axis is Point E in Fig. 2d. $w_{y}$ is the longitudinal vorticity at Sec. B2 in 1977(a1), 1994(a2), 2003(a3), and 2010(a4). 
As a whole, over the study period, the longitudinal estuarine circulation continued to increase, whereas the lateral circulation experienced varying changes at different cross-sections. At the upstream cross-section (B1), when the estuary narrowed, the original pattern of two-cell vortices with opposite polarity was disrupted. However, it was amplified in 2010 when the water depth was increased. At the cross-section in the middle of the estuary (B2), a similar two-cell pattern was developed. However, in 2003 and 2010, the single cell at the West Shoal was split into two cells: an anticlockwise cell at the surface and a clockwise cell at the lower part.

\subsection{Relationship between the Changes in the intensity of estuarine circulation} and the changes in topography

To further quantitatively identify the influence of topographic changes on the estuarine circulation, we calculated the changes in the intensity of estuarine circulations in the longitudinal and lateral directions. The magnitude of estuarine circulation in the longitudinal section was used to represent the intensity of the longitudinal estuarine circulation (Chen and Sanford, 2009). The method was to subtract the subtidal longitudinal velocity of the bottom layer from that on the surface layer. The magnitude of the vorticity in the cross-sections was used to represent the intensity of the lateral circulation (Becherer et al. 2015), and is expressed as:

$$
w_{y}=\partial w / \partial x-\partial u / \partial z
$$

where, $w_{y}$ is the longitudinal vorticity in the cross-sections. $w$ and $u$ are the currents in the vertical and lateral directions, respectively. $\partial w / \partial x$ is much smaller and can be ignored, therefore, the formula for calculating the intensity of lateral circulation can be simplified as:

$$
w_{y}=-\partial u / \partial z
$$

when $w_{y}$ is positive, the lateral circulation is an anticlockwise vortex, conversely, when $w_{y}$ is negative, the lateral circulation is a clockwise vortex. 
4.

487

\begin{tabular}{|c|c|c|c|c|c|c|}
\hline & & time & $1977 / 03$ & 1994/03 & $2003 / 03$ & 2010/03 \\
\hline \multirow{6}{*}{ Sec.B1 } & \multicolumn{2}{|c|}{ width (km) } & 5.56 & 2.25 & 2.26 & 2.14 \\
\hline & \multicolumn{2}{|c|}{ depth (m) } & 7.58 & 8.76 & 8.50 & 13.73 \\
\hline & \multicolumn{2}{|c|}{ w-to-d } & 734 & 257 & 266 & 156 \\
\hline & \multicolumn{2}{|c|}{ area $\left(\mathrm{km}^{2}\right)$} & 0.0468 & 0.0213 & 0.0207 & 0.0256 \\
\hline & \multicolumn{2}{|c|}{ narrowing rate } & 1 & $59.50 \%$ & $-0.44 \%$ & $5.30 \%$ \\
\hline & \multicolumn{2}{|c|}{ deepening rate } & 1 & $15.58 \%$ & $-2.95 \%$ & $61.47 \%$ \\
\hline \multirow{6}{*}{ Sec.B2 } & \multicolumn{2}{|c|}{ width (km) } & 18.97 & 15.77 & 11.40 & 10.76 \\
\hline & \multicolumn{2}{|c|}{ depth (m) } & 5.25 & 5.40 & 5.12 & 6.13 \\
\hline & \multicolumn{2}{|c|}{ w-to-d } & 3610 & 2920 & 2230 & 1760 \\
\hline & \multicolumn{2}{|c|}{ area $\left(\mathrm{km}^{2}\right)$} & 0.0849 & 0.303 & 0.0647 & 0.0646 \\
\hline & \multicolumn{2}{|c|}{ narrowing rate } & 1 & $16.87 \%$ & $27.71 \%$ & $5.61 \%$ \\
\hline & \multicolumn{2}{|c|}{ deepening rate } & 1 & $2.86 \%$ & $-5.19 \%$ & $19.73 \%$ \\
\hline \multirow{3}{*}{$\begin{array}{l}\text { circulation } \\
\text { intensity }\end{array}$} & longitudinal & Sec. A & 0.0274 & 0.0428 & 0.0483 & 0.0594 \\
\hline & \multirow{2}{*}{ lateral } & Sec. B1 & 0.0111 & 0.0146 & 0.0130 & 0.0278 \\
\hline & & Sec. B2 & 0.0493 & 0.0460 & 0.0465 & 0.0425 \\
\hline
\end{tabular}

496

Table 4. The changes of width and depth (the maximum depth), area (cross-section area), w-to$\mathrm{d}$, narrowing rate, deepening rate, and the intensity of circulations (w-to-d: width-to-depth ratio; narrowing rate: the ratio of the difference of cross-section widths between two years divided by the width in the earlier year; deepening rate: the ratio of the difference of water depth in the cross-section between the corresponding two years divided by the earlier depth. The positive narrowing rate indicates that the estuary is narrowed; the positive deepening rate indicates that the estuary is deepened.)

Table 4 indicates that the longitudinal estuarine circulation intensity increased with the estuary narrowing, and reached largest in 2010, which was $0.0594 \mathrm{~m} / \mathrm{s}$.

The lateral circulation intensity varied in different cross-sections. For Sec.B1, it increased gradually when the estuary deepened (from 1994 to 2010). When the deepening rate reached the maximum $(61.47 \%)$ in 2010 , the lateral circulation intensity reached the maximum as well. The intensity of lateral circulation increased when the estuary deepened and narrowed (from 1977 to 1994, and from 2003 to 2010), but it decreased when the estuary shallowed and narrowed (from 1994 to 2003). For Sec.B2, 
505 the intensity of lateral circulation decreased when the estuary deepened and narrowed

506 (from 1977 to 1994 , and from 2003 to 2010). However, this trend was altered when the

507 estuary entered into the "narrowing and shallowing period", with the deepening rate

508 being $-5.19 \%$. The lateral circulation intensity increased in 2003 . The change in the

509 lateral circulation intensity indicates that changes in water depth were the dominant

510 factor affecting lateral circulation intensity.

511 In general, the relationship between the longitudinal estuarine circulation intensity and the estuary width showed a monotonic decrease, while that between the longitudinal estuarine circulation intensity and the water depth is a monotonic increase, but the lateral circulation intensity seemed to have no simple linear relationship with the topographic change, including changes in the estuary width, water depth, and crosssection area.

\section{Discussion}

\subsection{Contribution of momentum terms to the variation of the longitudinal} conducted a diagnostic study by examining the changes in terms of the momentum balance equations. We calculated each term of the momentum equation in the longitudinal direction in the tidally averaged timescale:

By comparing the changes in each term and linking them with the characteristics of morphological evolution, we try to explain the response of the longitudinal estuarine circulation to bathymetric change in the perspective of momentum balance. Though the

531 change in an individual momentum term in Eq. 4 can not represent the change in the

532 longitudinal estuarine circulation as a whole, it can reflect the change in the 533 corresponding component for the estuarine circulation (Cheng, 2013). For example, an 
increase or decrease in the baroclinic pressure gradient force can reflect the change in the gravitational circulation, and the change in the advection term is representative of the change in tidal rectification. In the following, we present both the vertically averaged and depth-dependent values for these different terms along the longitudinal section in different years. It should be noted that the friction term consists of a component of the tidally mean eddy viscosity multiplied by the tidally mean vertical current shear, and a component of the correlation between eddy viscosity and vertical current shear, which is referred to as the tidal straining (Simpson et al., 1990).

Figure 7 shows that during the neap tide, the baroclinic pressure gradient force was balanced by barotropic gradient force, friction, and advection term in each year. This is different from the classic estuarine momentum balance (Pritchard, 1956) but consistent with the recent understanding of estuarine physics (Geyer and MacCready, 2014). The Coriolis force is quite small as both the latitude of the HE and the residual current are small. The high value of the baroclinic term was observed to shift upstream over time. As the baroclinic term is the multiplication of the salinity gradient and water depth, the changes in this term over years can be induced by the change in water depth and/or the salinity gradient. It can be seen from Fig. 4 that in the north of the null point, the salt intrusion gradually moved towards the bayhead with the estuary narrowing, thus increasing the salinity gradient there. In the meantime, the upstream water depth was increased due to channel dredging, particularly in 2010. Therefore, the increase of the baroclinic force term was caused by both the increases in water depth and salinity gradient. Although the barotropic term contributed a lot to the momentum balance, it did not change obviously with the morphological evolution. The advection term at the upstream section (B1) increased slightly with the estuary narrowing, especially in the deepening part of the channel in 2010. The friction term at the upstream section (B1) was the largest in 2010, because the salt intrusion increased the vertical shear of the longitudinal current there. Nevertheless, the increase in friction term was much smaller than that of the baroclinic term. Chant et al. (2018) attributed the increase in exchange 
563 by deepening, but in our case, the increase in baroclinic term was dominant and the

564 change in vertical mixing even posed a reversed effect.
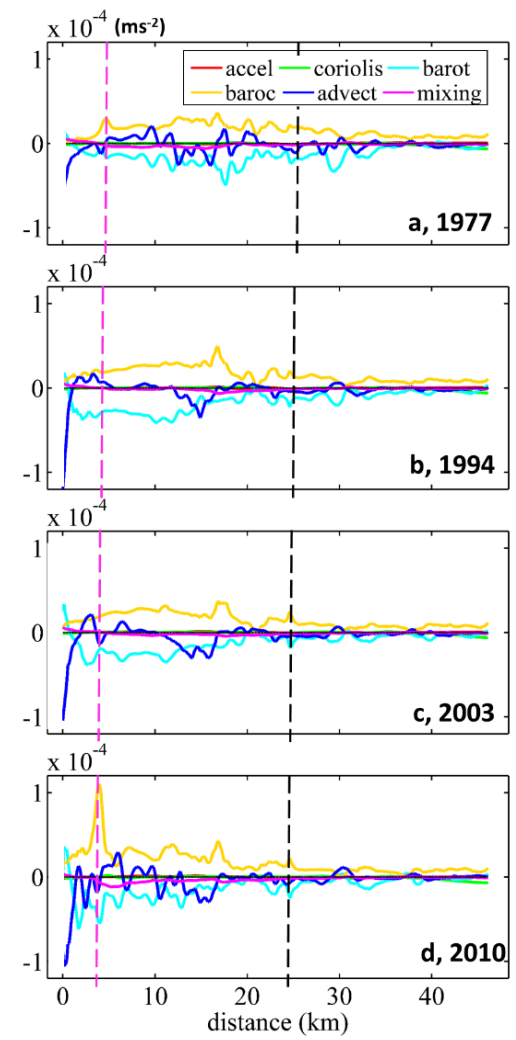

Fig. 7. Patterns of the longitudinal momentum terms during neap tide at Sec. A in 1977(a), 1994(b), 2003(c), and 2010(d). The starting point of the X-axis is Point A in Fig. 1b. "accel" in legend: local acceleration term; "barot" in legend: the barotropic gradient force term; "baroc" in legend: the baroclinic gradient force.

In Fig.8, we present the distribution of each momentum term in the longitudinal section and its change with the morphological evolution. Among them, the distribution patterns in 1994 and 2003 were very similar. The area of maximum bottom landward pressure gradient force was shifted to the upper bay. The magnitudes of the bottom pressure gradient force in 1994 and 2003 were greater than that in 1977, but less than that in 2010. The advection term alternated between positive and negative along Sec. A, and was increased in the upper part of Sec. A in 2010. The friction term generally 
578 shows a pattern of positive in the upper part and negative in the lower part of the water

579 column, opposite to the estuarine circulation. In the region where the seaward flow was

580 dominant in the whole water column (around $30 \mathrm{~km}$ ), the friction was positive near the

581 bottom. It can be seen that near Sec. B1, the friction at the bottom increased in 2010,

582 showing an increase in the bottom landward flow.
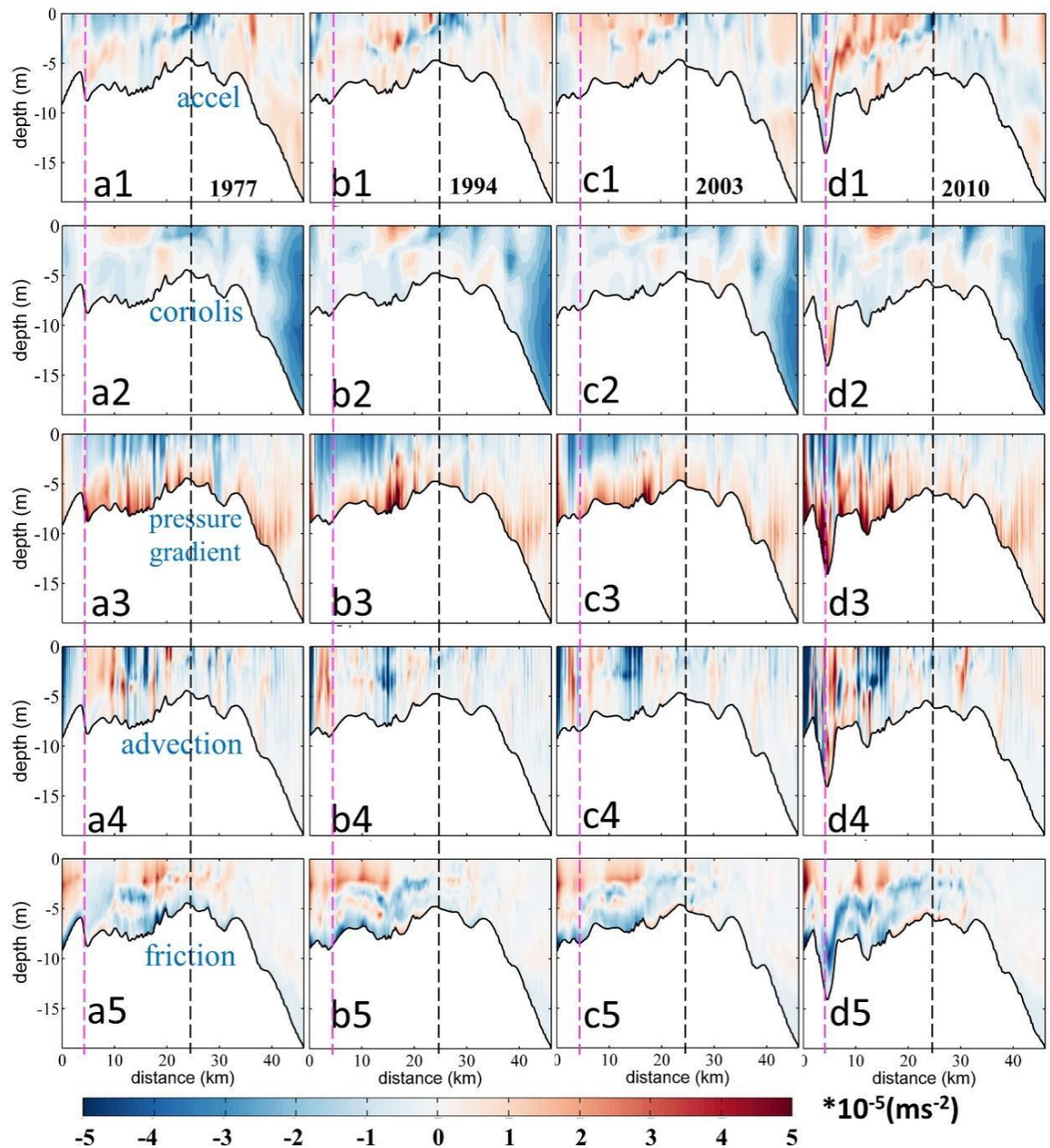

Fig. 8. Patterns of the longitudinal momentum terms during neap tide at Sec. A. (a1-d1): The local acceleration term. (a2-d2): The Coriolis term. (a3-d3): The pressure gradient force term. (a4-d4): The advection term. (a5-d5): The friction (vertical mixing term). 1977, 1994, 2003, and 2010 cases are in the first, second, third, and fourth columns, respectively. The pink and 
of the $\mathrm{X}$-axis is Point A in Fig. 1b. For viewing purposes, the acceleration term is multiplied by 10.

To further identify the changes in different terms, the advection term was divided into lateral (X-direction), longitudinal (Y-direction), and vertical (Z-direction) advection terms (Fig. 9). The sum of the advection terms in $\mathrm{X}$ and $\mathrm{Z}$ directions represents the effect of the lateral circulation. The lateral and longitudinal advection terms are collectively referred to as the horizontal advection term.
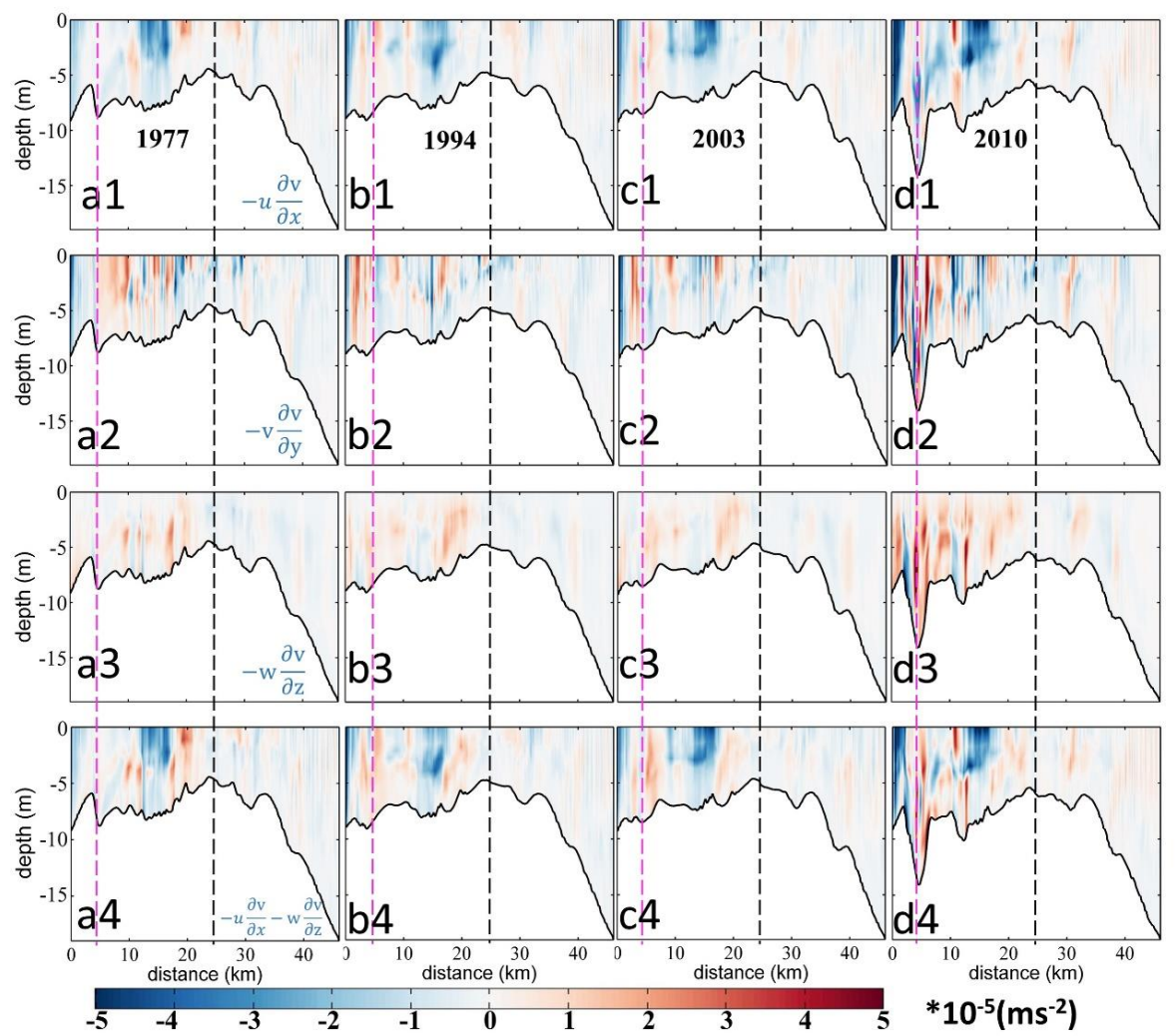

Fig. 9. Patterns of the longitudinal momentum terms during neap tide at Sec. A. (a1-d1): The advection in the $\mathrm{X}$ direction, $-u \frac{\partial v}{\partial x}$. (a2-d2): The advection in the $\mathrm{Y}$ direction, $-v \frac{\partial v}{\partial y}$. (a3-d3):

The advection in the $\mathrm{Z}$ direction, $-w \frac{\partial v}{\partial z}$. (a4-d4): The sum of the advection terms in $\mathrm{X}$ and $\mathrm{Z}$ directions. 1977, 1994, 2003, and 2010 cases are in the first, second, third, and fourth columns, respectively. The pink and black dotted lines represent the location of Sec.B1 and Sec.B2, respectively. The starting point of the $\mathrm{X}$-axis is Point A in Fig. $1 \mathrm{~b}$. 
From Fig. 9, in 2010, the advection terms in all directions increased significantly.

Generally, the lateral and vertical advection competes against each other, and their additive effect is to generate a circulation similar to the gravitational circulation. This effect was the strongest in 2010 (Figs. 9a4-d4). The longitudinal advection increased in the upper part of the channel in 2010 (Figs. 9a2-d2), following the deepening and narrowing of the estuary. In the middle of the longitudinal section, it induced a seaward flow at the surface and a landward flow at the bottom, whereas at the upper part, it generates a uniformly landward flow.

Therefore, the maximum longitudinal estuarine circulation in 2010 was caused by the increase in the pressure gradient force and the advection term, especially the baroclinic pressure gradient force. The largest variation of the advection and pressure gradient terms occurred in the period of topography narrowing and deepening.

Overall, from 1977 to 2010, the baroclinic force, the friction, and the advection terms were seen to increase along the Sec. A. We will further discuss the effects of these changes on estuarine circulation.

\subsection{Analysis of the streamwise vorticity balance for the lateral flow}

In order to reveal the contribution of the vertical shear of the along-channel flow, the lateral salinity gradient, and the vertical diffusion to changes in the lateral circulation, we examine the changes in terms of the streamwise vorticity transport equation (Li et al., 2014):

$$
\frac{d w_{y}}{d t}=\underbrace{-f \frac{\partial v}{\partial z}}_{\text {tilting of planetary }} \underbrace{-g \beta \frac{\partial S}{\partial x}}_{\text {vorticity baroclinicity }} \underbrace{+\frac{\partial^{2}}{\partial z^{2}}\left(K_{V} w_{y}\right)}_{\text {vertical diffusion }} \underbrace{+\frac{\partial^{2}}{\partial x^{2}}\left(K_{H} w_{y}\right)}_{\text {horizontal diffusion }}
$$

In the right side of Eq. 5, the first term represents the tilting of the planetary vorticity by vertical shear in the along-channel flow, the second term is the baroclinicity caused by the lateral salinity gradient, the third is the vertical diffusion, and the fourth is the horizontal diffusion, which is typically two orders of magnitude smaller than the vertical diffusion term. Therefore, we only show the first four terms in Fig. 10. 

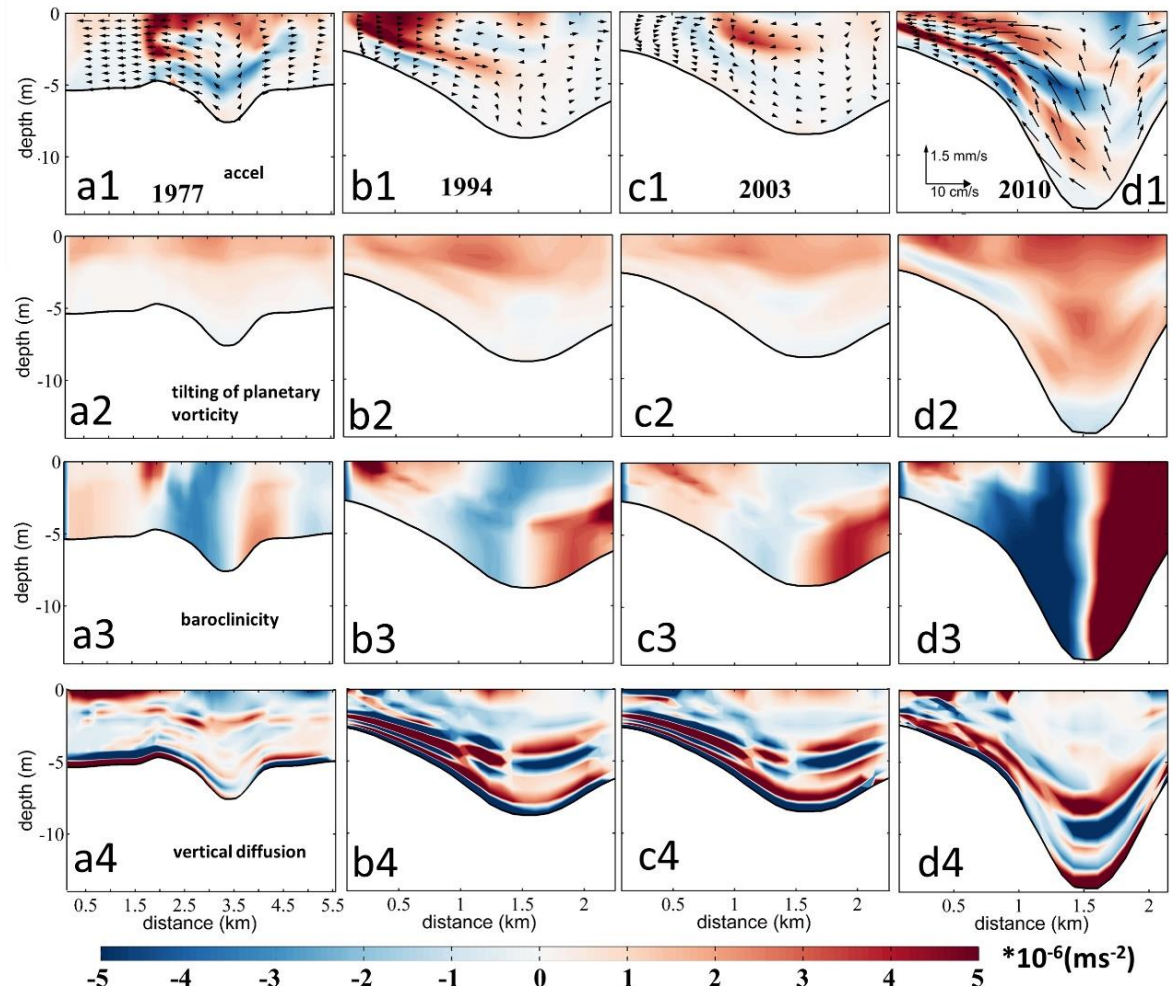

Fig. 10. Patterns of the streamwise vorticity equation terms during neap tide at Sec. B1. (a1$\mathrm{d} 1)$ : The local acceleration term. (a2-d2): The tilting of planetary vorticity term. (a3-d3): The baroclinicity term. (a4-d4): The vertical diffusion term. The cases in 1977, 1994, 2003, and 2010 are in the first, second, third, and fourth columns, respectively. The starting point of the $\mathrm{X}$-axis is Point $\mathrm{C}$ in Fig. 2d. For viewing purposes, the acceleration term is multiplied by 5 . The block arrows in a1-d1 represent the distribution of lateral circulation.

Figure 10 shows that the changes of baroclinicity terms caused by the water depth change dominated the changes in the lateral circulation at Sec. B1. The baroclinicity term in the deep channel was generally negative at the left side of the channel, and it increased significantly in 2010, about 2-3 times the value in 1977 . The baroclinicity term with positive values occurred over the West Shoal over the study period, but the areal extent occupied by the positive values decreased gradually, with its magnitude increased obviously in 1994 when the narrowing rate was the largest. A negative baroclinicity term appeared at the bottom of the West Shoal, indicating that the changes 
648 in water depth can lead to changes in the pattern and magnitude of the baroclinicity

649 term, which was mainly caused by the changes in the salt intrusion. The tilting of the

650 planetary vorticity term increased with the estuary narrowing, but the increase of this

651 term in 2010 was greater, which was mostly caused by the depth change. The pattern

652 of the vertical diffusion term changed significantly in 1977 and 1994. It changed at the

653 surface and the bottom layers of the West Shoal: When the width decreased to about

$6542.5 \mathrm{~km}$, the distribution of vertical diffusion was reversed compared with 1977,

655 indicating that it was the changes in width that altered the vertical diffusion term.
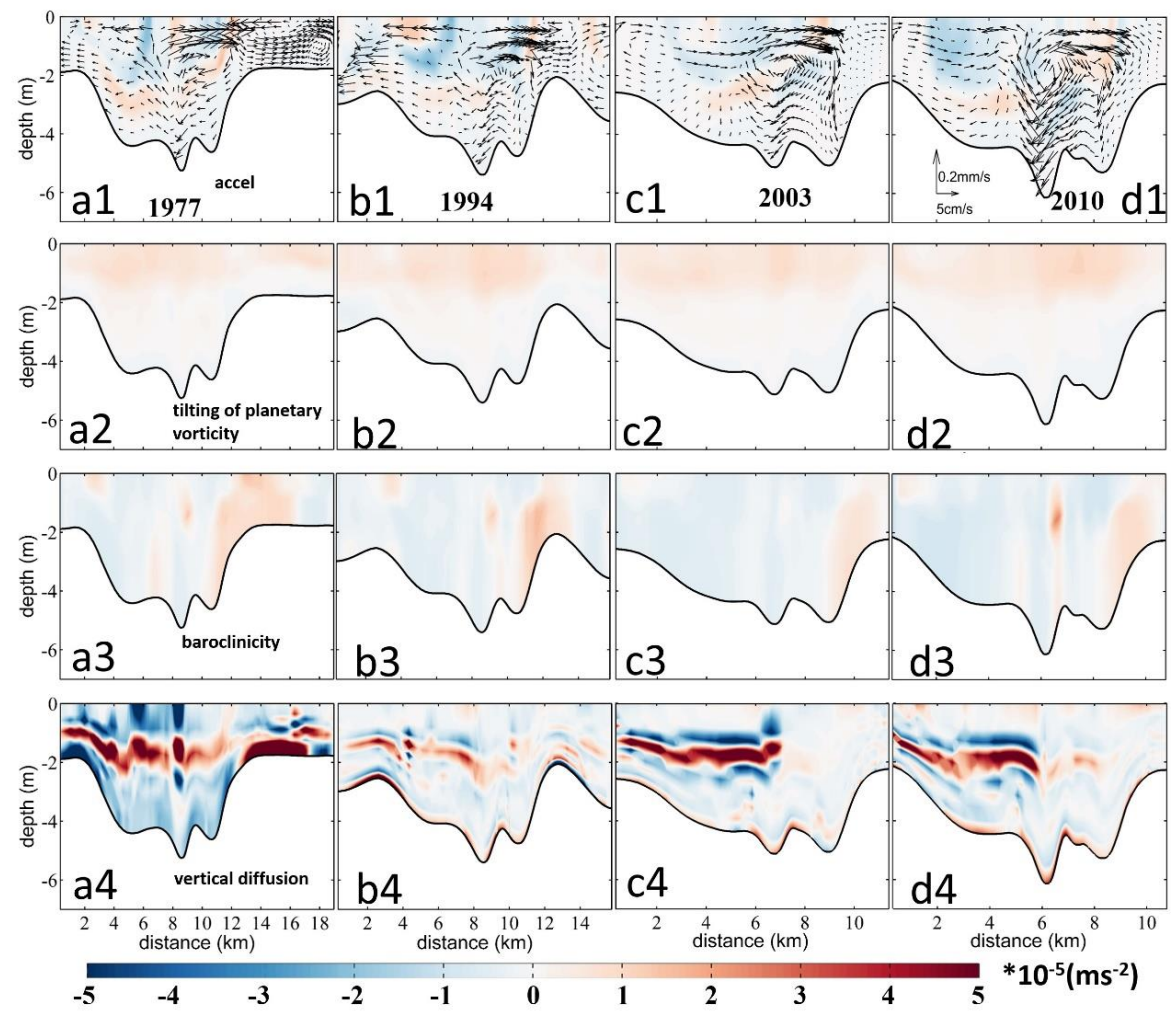

Fig. 11. Patterns of the streamwise vorticity equation terms during neap tide at Sec. B2. (a1d1): The local acceleration term. (a2-d2): The tilting of planetary vorticity term. (a3-d3): The baroclinicity term. (a4-d4): The vertical diffusion term. The cases in 1977, 1994, 2003, and 2010 are in the first, second, third, and fourth columns, respectively. The starting point of the $\mathrm{X}$-axis is Point $\mathrm{E}$ in Fig. 2d. For viewing purposes, the acceleration term is multiplied by 5 . The 
From Fig. 11, the change in the tilting of the planetary vorticity at Sec. B2 was analogous to that at Sec. B1. The baroclinicity term did not change much, because the changes in water depth were smaller than that at Sec. B1. The clockwise circulation over the West shoal increased as the estuary deepened in 2010, because the baroclinicity term was larger with the increase of salt intrusion and vertical salinity gradient near Sec. B2. The vertical diffusion of the vorticity was overall negative, indicating its effect in dissipating the vorticity. The vertical diffusion term was larger than the baroclinicity term, especially in the middle water, which was inconsistent with the conclusion that the baroclinicity term is the most important one in the lateral circulation (Li et al., 2014). The reason may be that in our study site, the vertical mixing was strong as the estuary was shallow. However, the existence of a pycnocline greatly weakened the momentum exchange between the upper and lower layers: above the pycnocline, the tilting of the planetary vorticity was dominant; whereas, under the pycnocline, the baroclinic term was dominant. The decrease of the estuary width changed the magnitude and pattern of the vertical diffusion term, especially when the estuary width was less than $15 \mathrm{~km}$, the area with a large positive value at the bottom of the East Shoal disappeared, and the magnitude of the negative value decreased greatly at the easternmost of the section. It indicates that in a shallow estuary, the vertical diffusion term caused by the width change is also important.

In Summary, the tilting of the planetary vorticity increased with the decrease of width or with the increase of water depth. The variation of estuary width was responsible for the changes in the vertical diffusion term, and the changes in water depth were responsible for the changes in the baroclinicity term. The increase of the longitudinal estuary circulation can increase the baroclinicity term of the cross-sections by increasing the salinity gradient near the cross-sections, which mainly occurred in the periods of the estuary deepening. The deepening rate of Sec.B1 was the highest $(61 \%)$ in 2010, which led to the strongest lateral circulation in 2010. The lateral circulation

690 intensity decreased when the estuary narrowed in 2003 due to the decreased 691 baroclinicity term. In addition, the shallowing was the main reason for the pattern 
change of the lateral circulation at Sec.B2. At Sec. B2, the narrowing rate was the largest in 2003, and the adjustment of vertical diffusion term resulted in an increased lateral circulation from 1994 to 2003 . The decrease of the clockwise circulation at the East Shoal was mainly related to the adjustment of the vertical diffusion term to the baroclinicity term.

\subsection{Comparison to theoretical results and other estuaries influenced by human interventions}

The longitudinal estuarine circulation is generated by the river discharge, Stokes return flow, longitudinal baroclinic pressure force, tidal straining, and advection (Geyer and Maccready, 2014). The HE features a microtidal tidal regime (tidal range less than $1.5 \mathrm{~m}$ ), and the component generated by the baroclinic pressure gradient, i.e., the gravitational circulation, would be a primary part of the longitudinal estuarine circulation. The convergent geometry makes it susceptible to the residual flow induced by the longitudinal advection (Burchard et al., 2014). However, as seen above, the horizontal advection also plays a role in generating the estuarine circulation.

With channel deepening and width narrowing in the HE, the gravitational circulation was increased by the increased baroclinic pressure gradient force. Based on Geyer (2010), the gravitational circulation in a straight estuary of rectangular crosssection is scaled as:

$$
v_{g}=a\left(\beta g s_{0} h\right)^{1 / 5} U_{r}^{1 / 5} U_{t}^{2 / 5}=a\left(\beta g s_{0} h\right)^{1 / 5} R^{1 / 5}(w h)^{-1 / 5} U_{t}^{2 / 5}
$$

in which $a$ is a constant, $\beta$ is the salinity expansion coefficient, $g$ is the gravity acceleration, $s_{0}$ is the oceanic salinity, $R$ is the river discharge, $w$ is the width of the cross-section, $U_{t}$ is the tidal velocity amplitude, $h$ is the water depth, $U_{r}$ is the outflow velocity associated with the river discharge. Where $u_{r}=Q_{r} / A_{C S}\left(\mathrm{Q}_{r}\right.$ is the freshwater outflow, $\mathrm{A}_{C S}$ being the local cross-sectional area of the estuary). 
If we simply assume that the change in tidal current amplitude follows the Green's law $U_{t}=U_{0} \sqrt{\frac{w_{0} h_{0}}{w h}}$ (here $w_{0}$ and $h_{0}$ is the width and depth at the estuary mouth, respectively), then the gravitational circulation becomes: and width in the estuary, with a weaker dependence on the water depth. In Chant et al. (2018), the gravitational circulation is completely unrelated to the water depth in their equation (2), which is $v_{g} \propto\left(\frac{g^{\prime} R}{w}\right)^{\frac{1}{3}}$, in which the $g^{\prime}$ is the reduced gravity acceleration. This seems to contradict the situations occurring in many estuaries, such as in the Coos Bay (Eidam et al., 2020), Tampa Bay (Zhu et al., 2015), Changjiang Estuary (Zhu, 2018), Ems estuary (Van Maren et al., 2015), Hudson Estuary (Ralston and Geyer, 2019), and Newark Bay of the Delaware estuary (Chant et al., 2018). In all these estuaries, the gravitational circulation demonstrated an increase with the deepening of the channel. It suggests that the changes in gravitational circulation vary in different parts of the estuary and the longitudinal salinity gradient may not catch up with the change in water depth in the analytical solution, proposed by Chant et al. (2018) and Ralston and Geyer (2019). In our study site, the salinity gradient at the upstream part of the longitudinal section was increased owing to an enhanced salt intrusion where water depth increased, which led to an increased gravitational circulation in the upstream of the HE (Fig. 4).

The tidal straining-induced estuarine circulation is another important component of longitudinal estuarine circulation. The straining-induced circulation is the covariance of the eddy viscosity and the vertical shear of the longitudinal flow (ESCO) in a tidal cycle and is included in the term of internal friction. Cheng et al. (2010) have indicated that ESCO-induced flow dominates the gravitational circulation in periodically stratified estuaries with strong tides, having the same structure as the gravitational 
circulation. It has the same order of magnitude in weakly stratified estuaries with moderate tides, and is less important in highly stratified estuaries with weak tides, even with a reversed structure with the gravitational circulation. As indicated by Becherer et al. (2015), the strength of the straining-induced circulation is dependent on the Simpson number (or the horizontal Richardson number). The Simpson number is expressed as:

$$
S_{i}=g \beta \frac{d S}{d y} \frac{h^{2}}{u_{*}},
$$

in which $u_{*}$ is the bottom friction velocity, represented by $u_{*}=\sqrt{C_{d}} U_{t}$, where $C_{d}$ is the bottom friction coefficient and $U_{t}$ is the tidal velocity amplitude.

When $S_{i}$ lies in the range of 0.088 to 0.84 , the water column stays periodically stratified, and the straining-induced circulation is an important component in the longitudinal estuarine circulation. When $S_{i}$ is larger than 0.84 , the water column is in a persistent stratified situation, and the straining-induced circulation becomes weaker. We calculated the $S_{i}$ along the longitudinal section in different years and depict them in Fig. 12.

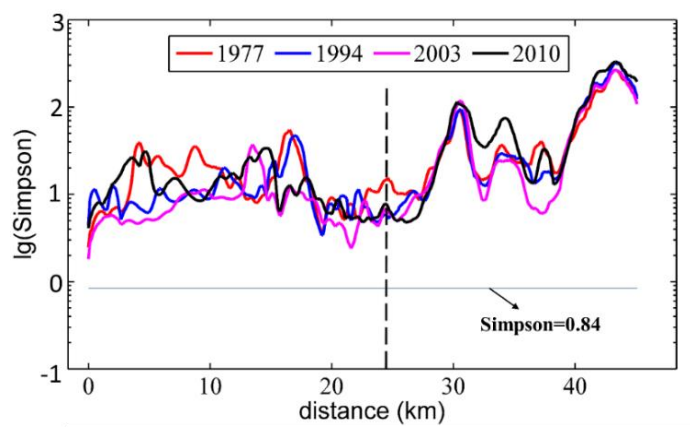

Fig. 12. Distribution of the Simpson number in different years along the longitudinal section. The Y-axis represents the logarithmic of the $S_{i}$. The black dotted line represents the location of the null point.

It indicates that along the longitudinal section, the $S_{i}$ number was mostly above the criterion of 0.84 , showing that the straining-induced circulation is not significant. The Si number was the smallest in 2003 and the largest in 2010. It indicates that with the narrowing and deepening of the HE, the straining-induced circulation became 
weaker. This is consistent with Burchard et al. (2014) and Schulz et al. (2015). It indicates that with the human interventions, the straining-induced circulation became less important in the longitudinal estuarine circulation.

For the advection-induced longitudinal estuarine circulation, we noted that the longitudinal and vertical advection terms were smaller than the lateral advection. Based on Cheng and Valle-Levinson (2009), the lateral advection-induced longitudinal circulation is proportional to the ratio of $h /\left(w K_{m}\right)$, where $w$ is the width, and $K_{m}$ is the eddy viscosity. It shows that in a narrower and deeper estuary, the lateral advection has a larger effect in influencing the longitudinal estuarine circulation. Lerczak and Geyer (2004) also showed that the effect of the lateral advection on longitudinal circulation is stronger for narrower estuaries. Our results show that with the narrowing and deepening of the estuary, not only the lateral advection but also the longitudinal advection has great influences on the longitudinal estuarine circulation.

\subsection{The possible future development of the estuarine circulation and its}

\section{implications}

The pattern of lateral circulation during the dry season in the HE experienced a dramatic change from 2003 to 2010 in the West Shoal at Sec. B2, from an underdeveloped circulation structure to a complete clockwise vortex in 2010. This transition was associated with the increase in lateral salinity gradient, the increase in longitudinal bottom landward flow, and a decrease of friction by the increased water depth and stratification.

The mechanisms for the lateral circulation during the wet season have been revealed by Chen et al. (2020b), who showed that it was primarily driven by the barotropic process, i.e., the water elevation gradient, and thus by the intensity of the ebb jet. Different from the wet season when the river discharge was more dominant, the lateral circulation in the dry season was more affected by the baroclinic effect. We 
even in the wet season will be enhanced with the ebb jet in the deep channel strengthened.

In the HE, the channel underwent siltation, and sediment was carried from the channels to side banks by the lateral circulation, making the estuary overall shallower in 2003. In 2005, dredging of the channel increased the channel depth (Luo, 2010), resulting in a high deepening rate, and increased the longitudinal estuarine circulation, though the lateral circulation decreased slightly by the smaller rate of convergence. If reclamation did not occur as frequently as it did in the last century, and the channel dredging continued, the estuarine circulation of the estuary will in general keep increasing with the increase in water depth, and there exists positive feedback. However, as revealed in Eq. (6) and Eq. (2) in Chant et al. (2018), with the increase in salt intrusion, the longitudinal salinity gradient will decrease, showing negative feedback. Moreover, Schulz et al. (2015) noted that estuarine circulation exhibits a distinct maximum in medium-wide channels by comparing estuarine circulation under different width-to-depth ratios. In our study, as shown in Table 4, the width-to-depth ratio has been decreasing from 1977 to 2010, but the estuarine circulation has been increasing. The difference would be caused by the fact that in our study site, the tidal mixing is not strong enough to generate an effective tidal straining-induced circulation.

The changes in the estuarine circulation have important implications for sediment transport and morphological evolution in the HE. With the increase of longitudinal estuarine circulation, the sediment trapping effect is expected to be enhanced, thus more riverine sediment would be trapped inside the estuary. In the meantime, the change in lateral circulation would decrease the sediment advection from the channel to the West Shoal, which occurred in the wet season and was favorable for the siltation in the West Shoal (Chen et al., 2020b). considering the effects of winds, waves, and other upstream flows into the estuary. Future work could incorporate the above factors to improve the model's accuracy. 


\section{Conclusion}

828

This study investigated the morphological evolution of the HE from 1977 to 2010 using ArcGIS and remote sensing. It was noted that the West Channel of the HE disappeared, causing the morphological pattern to change from "two channels and three shoals" gradually to "one channel and two shoals" throughout the years. Due to the reclamation and development of salt marshes along the estuarine banks, the estuary has been experiencing continuous narrowing. Meanwhile, channel dredging has deepened the estuary over the study period. The intensity of the longitudinal estuarine circulation kept increasing as the estuary width continued to decrease. The trend of the lateral circulation intensity altered when the estuary shallowed (from 1994 to 2003).

The changes in the longitudinal estuarine circulation were dominated by the changes in the baroclinic pressure gradient force and advection. As the estuary was narrowing and deepening, the pressure gradient force and advection term (especially the horizontal advection term) increased, which increased the longitudinal circulation. The change in lateral circulation intensity was mainly caused by the change of the vertical shear of the longitudinal subtidal flow, the lateral salinity gradient, and the vertical dissipation term. The changes in water depth were the dominant factor affecting lateral circulation intensity. The increase of water depth enhanced the longitudinal circulation and the lateral circulation of the upstream cross-section in 2010. The changes in the estuarine circulation have great implications for the sediment transport in the HE, which would be explored in the next step.

\section{Data availability}

A total of $142 \mathrm{G}$ data of 66 images (Table 1) covering the PRD during cloudless days in multiple years (from 1973 to 2018) were downloaded from http://www.gscloud.cn/. 
https://doi.org/10.5194/os-2021-76

Preprint. Discussion started: 6 September 2021

(c) Author(s) 2021. CC BY 4.0 License.

\section{Author contributions}

855 RuiZhang: Writing - original draft, model runs and analyses. Bo Hong: Writing -

856 review. Lei Zhu: Writing - review. Wenping Gong: Writing - review \& editing,

857 Conceptualization, Funding acquisition. Heng Zhang: Visualization, Funding

858 acquisition.

859

860

Competing interests

861 The authors declare that they have no conflict of interest.

862

863

Acknowledgments

864

This research is funded by the National Natural Science Foundation of China [Grant nos. 51761135021, 41506102, 41890851]. We would like to thank the National 
https://doi.org/10.5194/os-2021-76

Preprint. Discussion started: 6 September 2021

(c) Author(s) 2021. CC BY 4.0 License.

\section{Appendix A}
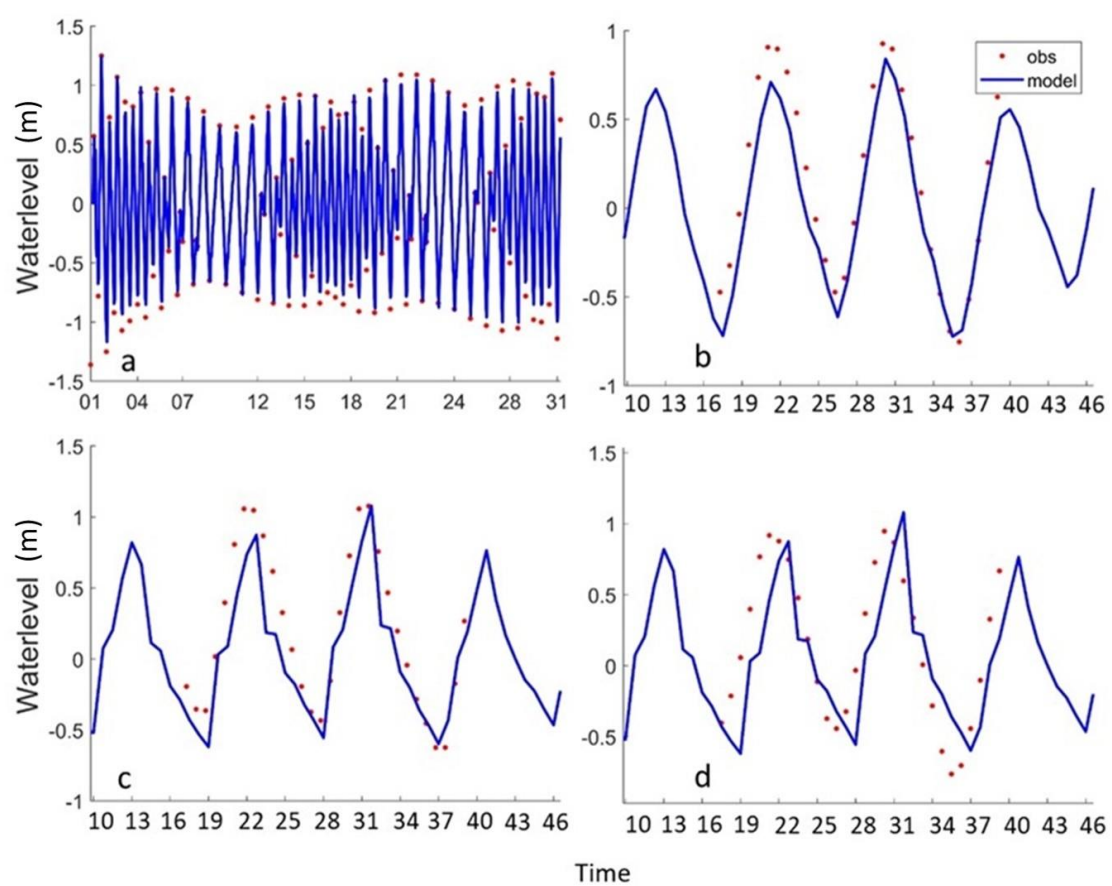

Fig. A. 1. Validation of water level of the MD2 model at 4 stations (S6 (a): SK=0.9969, DJ (b): $\mathrm{SK}=0.9337, \mathrm{~S} 1$ (c): $\mathrm{SK}=0.8652$, and GL (d): $\mathrm{SK}=0.8928$ ) in March 2010. The red dots and blue lines represent observation and simulated results, respectively. The station locations are shown in Fig. 1b. Time in (a) is days from 2010/3/1, in (b-d) is hours from 2010/3/17 00:00. 

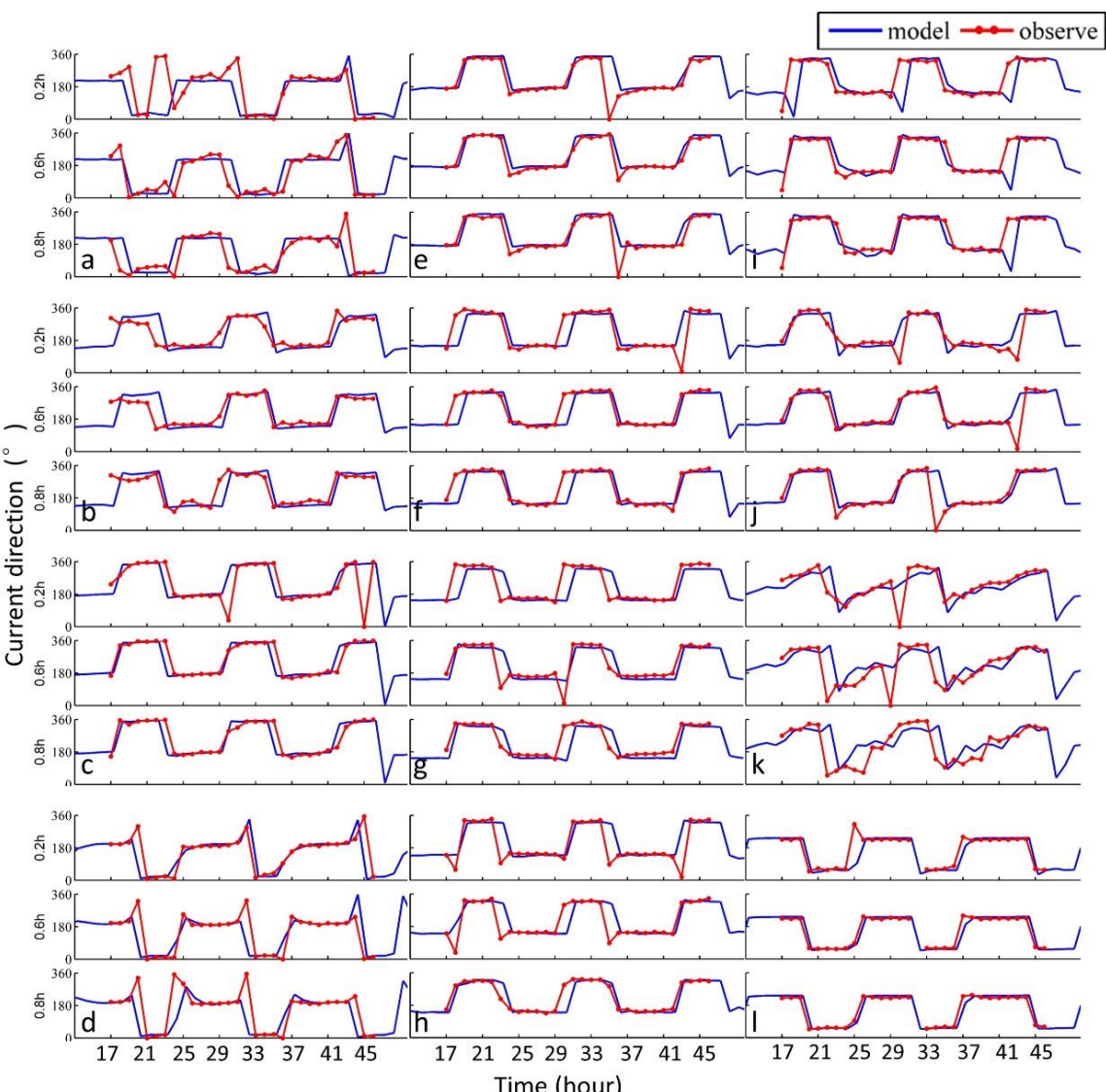

Fig. A. 2. Validation of current direction of the MD2 model at 12 stations (DJ (a), GL (b), HB (c), S2(d), S3(e), S4(f), S5(g), S7(h), S6(i), S8(j), S9(k), and S1(1)) in March 2010. The red and blue lines represent observation and simulation results, respectively. The station locations are shown in Fig. 1b. Time is hours from 2010/3/17 00:00. 


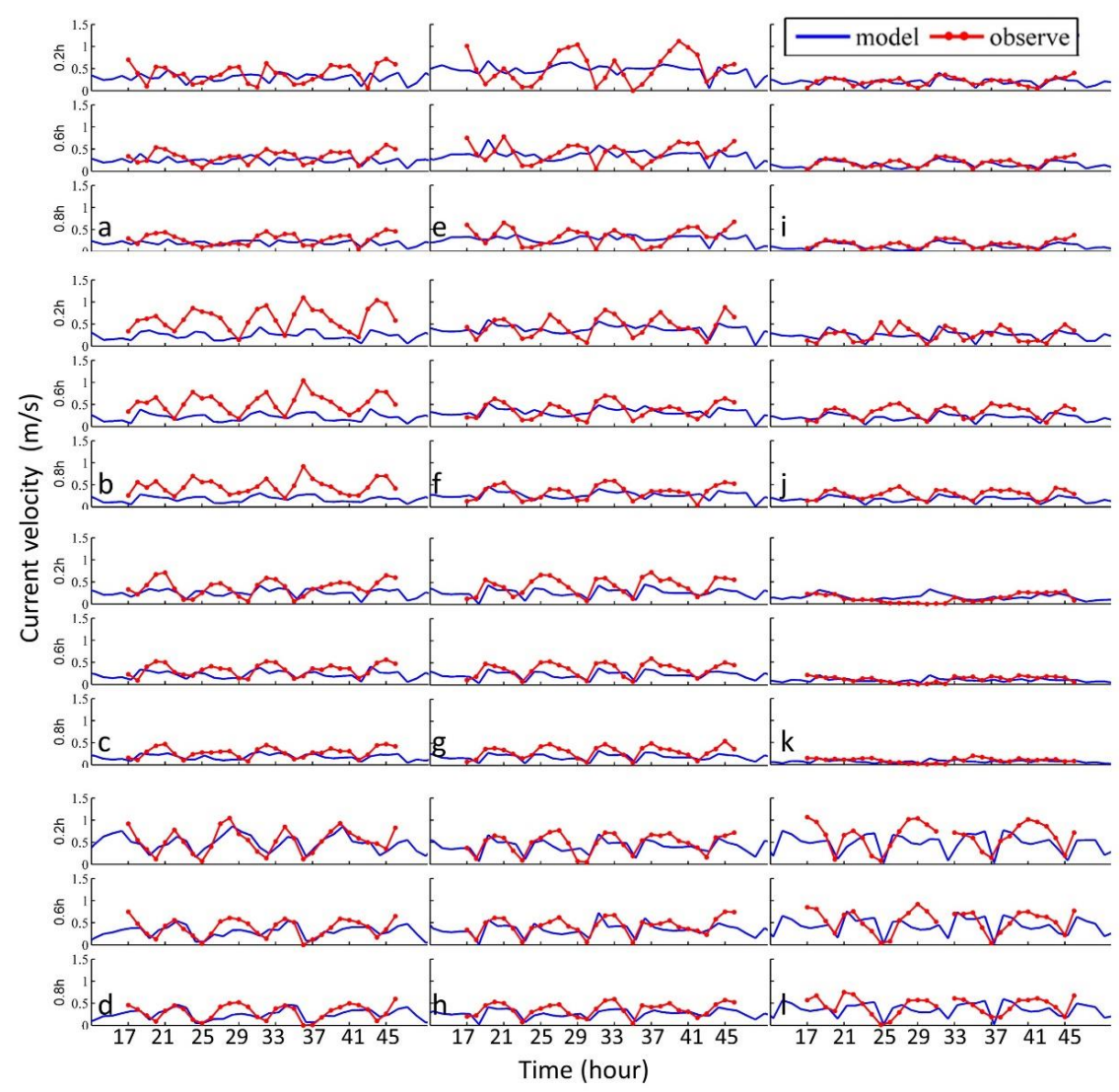

Fig. A. 3. Validation of current velocity of the MD2 model at 12 stations (DJ (a), GL (b), HB (c), S2(d), S3(e), S4(f), S5(g), S7(h), S6(i), S8(j), S9(k), and S1(1)) in March 2010. The red and blue lines represent observed and simulated results, respectively. The station locations are shown in Fig. 1b. Time is hours from 2010/3/17 00:00. 


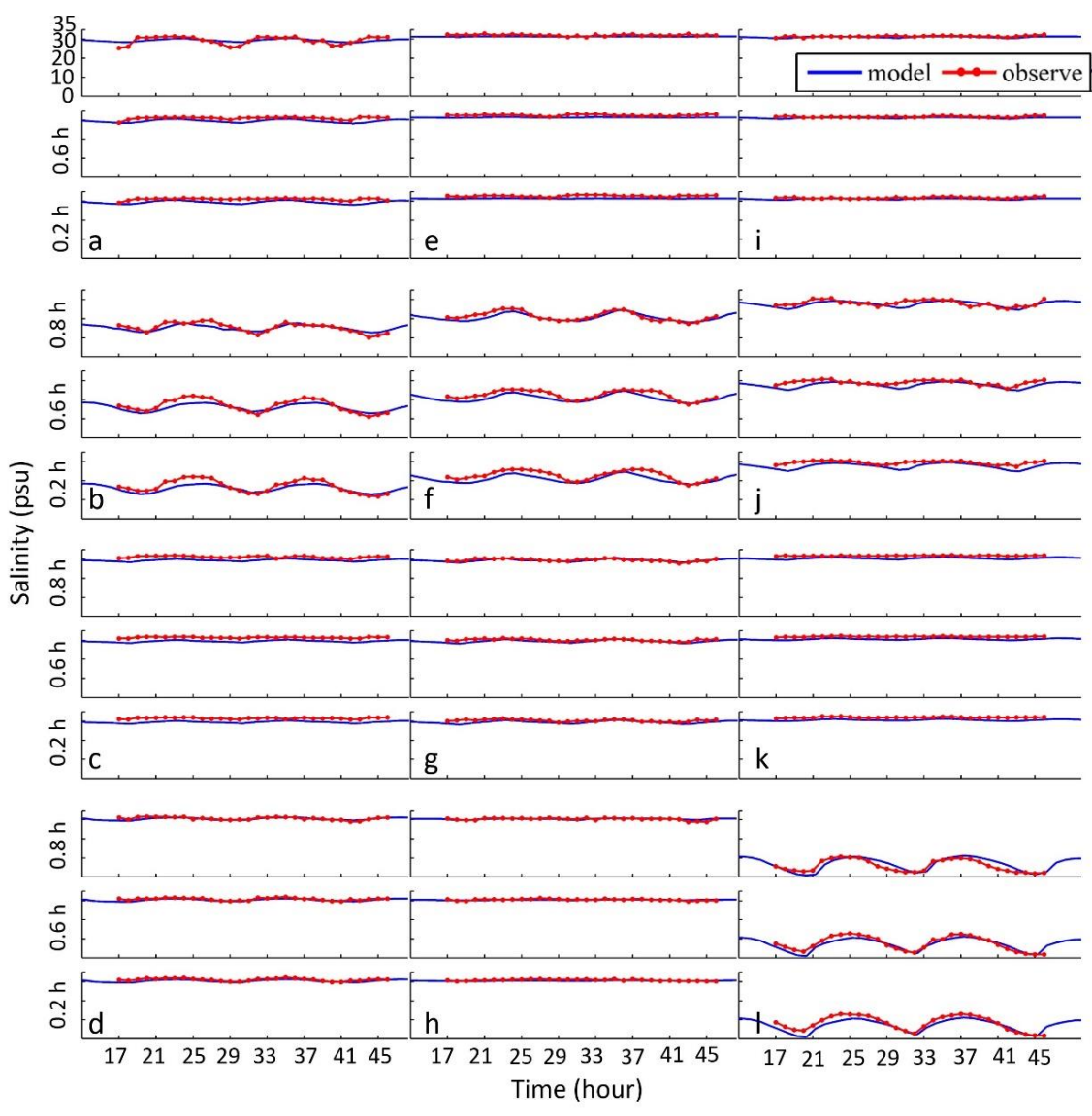

Fig. A. 4. Validation of salinity of the MD2 model at 12 stations (DJ (a), GL (b), HB (c), S2(d), S3(e), S4(f), S5(g), S7(h), S6(i), S8(j), S9(k), and S1(1)) in March 2010. The red and blue lines represent observed and simulated results, respectively. The station locations are shown in Fig. 1b. Time is hours from 2010/3/17 00:00. 


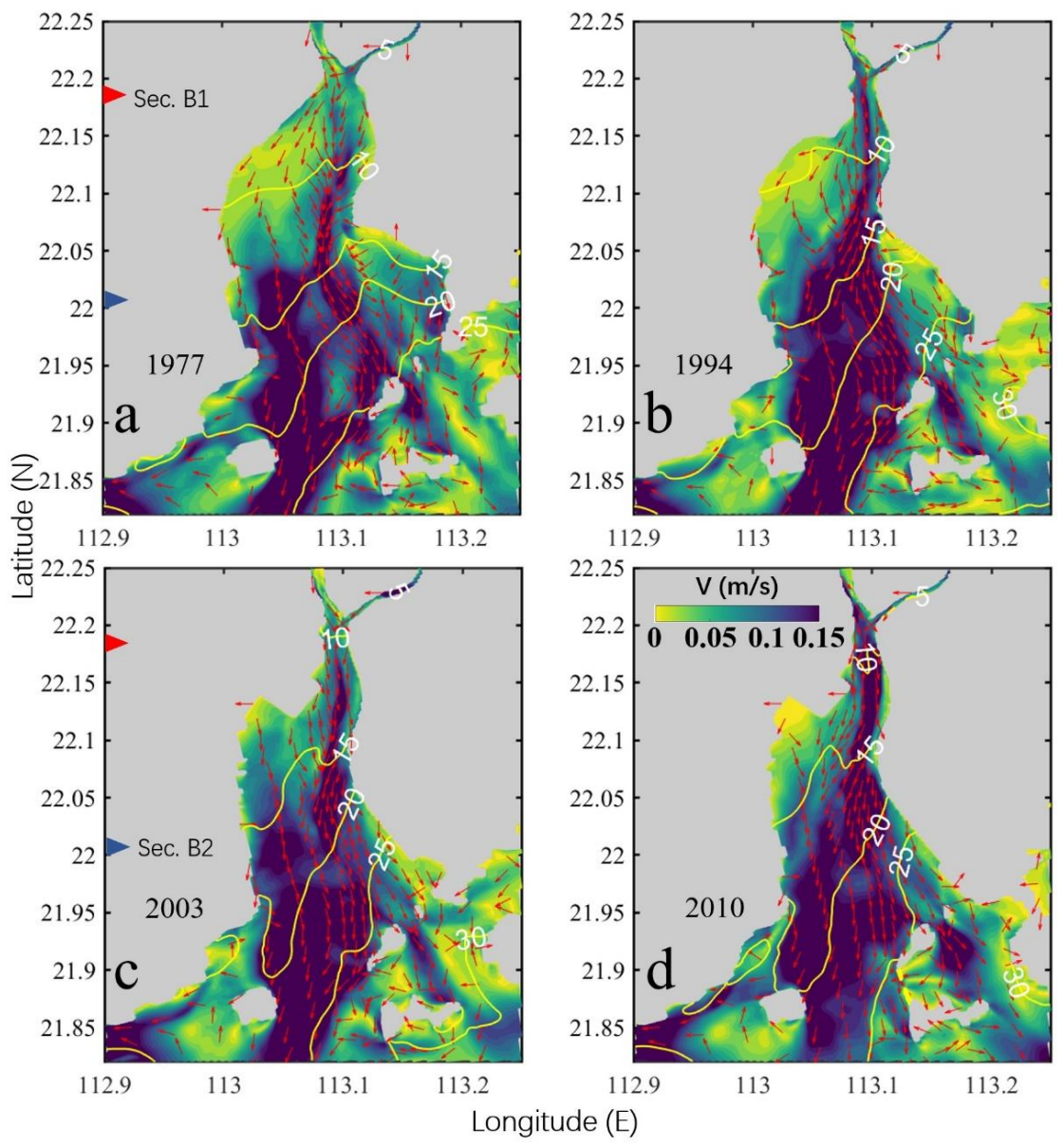

Fig. A. 5. Patterns of the horizontal circulation at the surface during neap tide in 1977(a1), 1994(a2), 2003(a3), and 2004(a4). The magnitude of the current is represented by the color shading, while the current direction is shown by the arrows. The salinity is depicted by the contour lines. The red and blue triangles depict the positions of two cross-sections (Sec.B1 and Sec.B2). 

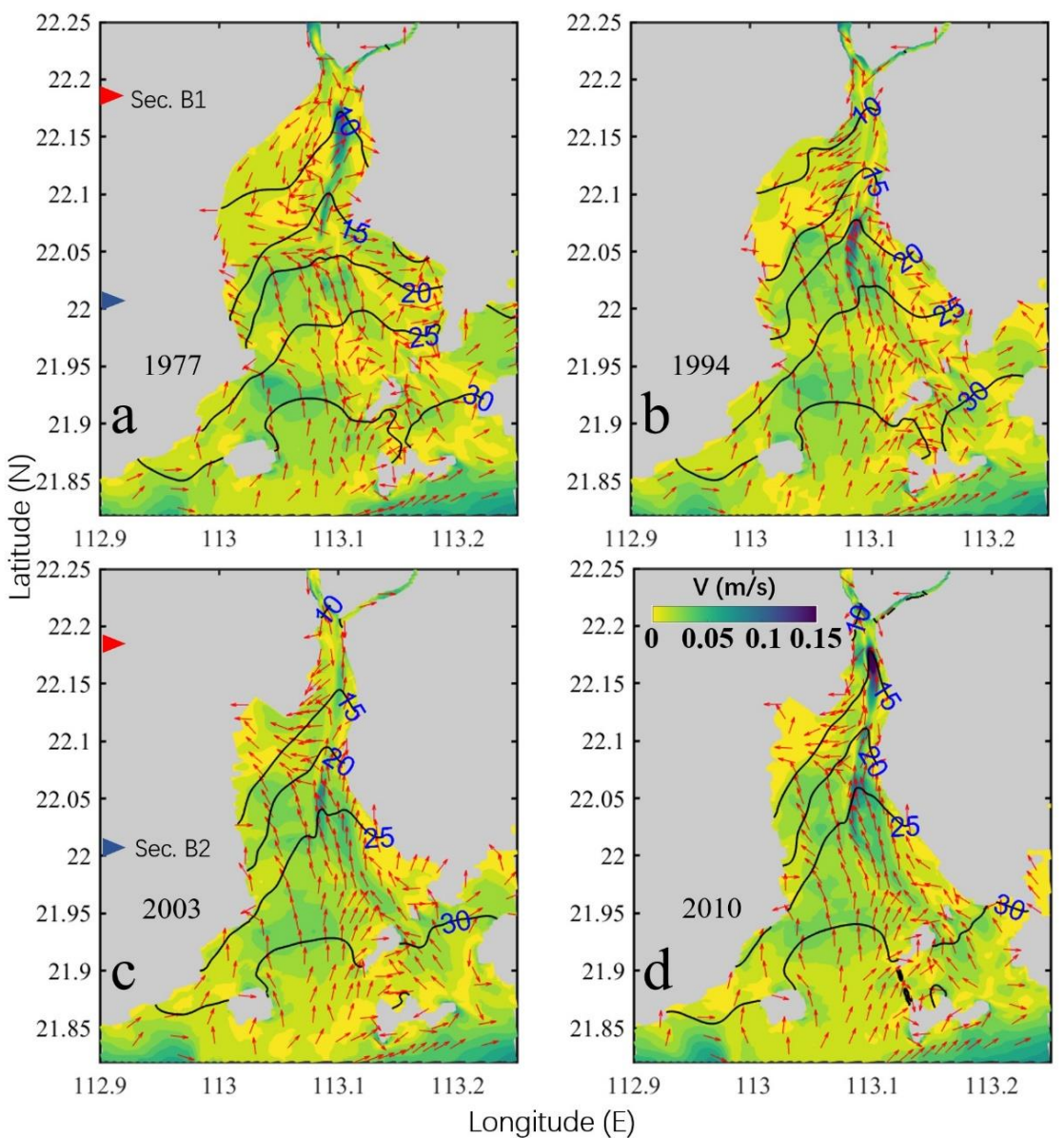

Fig. A. 6. Patterns of the horizontal circulation at the bottom during neap tide in 1977(a1), 1994(a2), 2003(a3), and 2004(a4). The magnitude of the current is represented by the color shading, while the current direction is shown by the arrows. The salinity is depicted by the contour lines. The red and blue triangles denote the positions of two cross-sections (Sec.B1 and Sec.B2). 


\section{References}

Ai, B., Zhang, R., Zhang, H., Ma, C. L. and Gu, F. G.: Dynamic process and artificial mechanism of coastline change in the Pearl River Estuary, Regional Studies in Marine Science, 30, 100715, 2019.

Amin, M.: On perturbations of harmonic constants in the Thames Estuary, Geophysical Journal of the Royal Astronomical Society, 73, 587-603, 1983.

Becherer, J., Stacey, M. T., Umlauf, L. and Burchard, H.: Lateral circulation generates flood tide stratification and estuarine exchange flow in a curved tidal inlet, J. Phys. Oceanogr., 45, 638-656, 2015.

Burchard, H., Hetland, R. D., Schulz, E. and Schuttelaars, H. M.: Drivers of Residual Estuarine Circulation in Tidally Energetic Estuaries: Straight and Irrotational Channels with Parabolic Cross Section, J. Phys. Oceanogr., 41, 548-570, 2010.

Burchard, H., Schulz, E. and Schuttelaars, H. M.: Impact of estuarine convergence on residual circulation in tidally energetic estuaries and inlets, Geophys. Res. Lett., 41, 913-919, 2014.

Chant, R. J., Sommerfield, C. K. and Talke, S. A.: Impact of channel deepening on tidal and gravitational circulation in a highly engineered estuarine basin, Estuar. Coast., 41, 1587-1600, 2018.

Chen, L. H., Gong, W. P., Scully, M. E., Zhang, H., Cheng, W. C. and Li, W.: Axial wind effects on stratification and longitudinal sediment transport in a convergent estuary during wet season, Journal of Geophysical Research: Oceans, 125, e2019J-e15254J, 2020a.

Chen, L. H., Gong, W. P., Zhang, H., Zhu, L. and Cheng, W. C.: Lateral circulation and associated sediment transport in a convergent estuary, Journal of Geophysical Research: Oceans, 125, e2019Je15926J, 2020b.

Chen, S. N. and Sanford, L. P.: Axial Wind Effects on Stratification and Longitudinal Salt Transport in an Idealized, Partially Mixed Estuary, J. Phys. Oceanogr., 39, 1905-1920, 10.1175/2009JPO4016.1, 2009.

Cheng, P.: Decomposition of Residual Circulation in Estuaries, Journal of Atmospheric \& Oceanic Technology, 31, 698-713, 2013.

Cheng, P. and Valle-Levinson, A.: Influence of lateral advection on residual currents in microtidal estuaries, J. Phys. Oceanogr., 39, 3177-3190, 2009.

Cheng, P., Valle-Levinson, A. and De Swart, H. E.: Residual currents induced by asymmetric tidal mixing in weakly stratified narrow estuaries, J. Phys. Oceanogr., 40, 2135-2147, 2010.

Chernetsky, A. S., Schuttelaars, H. M. and Talke, S. A.: The Effect of Tidal Asymmetry and Temporal Settling Lag on Sediment Trapping in Tidal Estuaries, Ocean Dynam., 60, 1219-1241, 2010.

Dyer, K. R. 1977. Lateral circulation effects in estuaries. National Academy of Sciences, p. 22-29.

Eidam, E. F., Sutherland, D. A., Ralston, D. K., Dye, B., Conroy, T., Schmitt, J., Ruggiero, P. and Wood, J.: Impacts of 150 Years of Shoreline and Bathymetric Change in the Coos Estuary, Oregon, USA, Estuar. Coast., 1-19, 2020.

Fischer, H. B.: Mixing and Dispersion in Estuaries, Annu. Rev. Fluid Mech., 8, 107-133, 10.1146/annurev.fl.08.010176.000543, 1976.

Geyer, W. R.: Estuarine salinity structure and circulation, Contemporary issues in estuarine physics, 12, 26, 2010.

Geyer, W. R. and Maccready, P.: The Estuarine Circulation, Annu. Rev. Fluid Mech., 46, 175-197, 2014.

Gong, W. P., Jia, L. W., Shen, J. and Liu, J. T.: Sediment transport in response to changes in river 

2014.

Gong, W. P., Liu, H., Ren, J. and Yu, H. B.: The study of tidal propagation in the Huangmaohai estuary and its underlying mechanisms, Acta Oceanol. Sin., 34, 41-54, 2012.

Gong, W. P., Schuttelaars, H. and Zhang, H.: Tidal asymmetry in a funnel-shaped estuary with mixed semidiurnal tides, Ocean Dynam., 66, 637-658, 2016.

Huang, T. 2011. Study on abnormal changes of tidal range in the huangmaohai estuary. Guangdong Water Resources and Hydropower, Guangzhou, China.

Jia, L. W., Luo, J. and Ren, J.: The analysis of the evolution of a sand bar and its formation in the Huangmao Bay in the Pearl River Delta, Acta Oceanol. Sin., 34, 120-127, 2012.

Kjerfve, B., Stevenson, L. H., Proehl, J. A., Chrzanowski, T. H. and Kitchens, W. M.: Estimation of material fluxes in an estuarine cross section: A critical analysis of spatial measurement density and errors 1, Limnol. Oceanogr., 26, 325-335, 1981.

Lacy, J. R., Stacey, M. T., Burau, J. R. and Monismith, S. G.: Interaction of lateral baroclinic forcing and turbulence in an estuary, Journal of Geophysical Research: Oceans, 108, 1-34, https://doi.org/10.1029/2002JC001392, 2003.

Lerczak, J. A. and Rockwell Geyer, W.: Modeling the Lateral Circulation in Straight, Stratified Estuaries*, J. Phys. Oceanogr., 34, 1410-1428, 2004.

Lesser, G. R., Roelvink, J. V., Van Kester, J. and Stelling, G. S.: Development and validation of a threedimensional morphological model, Coast. Eng., 51, 883-915, 2004.

Li, C. Y. and O'Donnell, J.: Tidally driven residual circulation in shallow estuaries with lateral depth variation, Journal of Geophysical Research Oceans, 102, 27915-27929, 1997.

Li, M., Cheng, P., Chant, R. J., Valle-Levinson, A. and Arnott, K.: Analysis of vortex dynamics of lateral circulation in a straight tidal estuary, J. Phys. Oceanogr., 44, 2779-2795, 2014.

Li, W., Shi, J. Z., Pu, X. and Hu, G. D.: Circulation within curved channel of the north passage in the changjiang river estuary: a vorticity approach, Oceanologia et Limnologia Sinica, 48, 682-694, 2017.

Li, Y. B. 2019. Numerical simulation of the formation and evolution of the geomorphic characteristics of Huangmao Sea. Dalian University of Technology, Dalian, China.

Luo, J. 2010. Cause Analysis of Morphological evolution of Huangmao sea Estuary in the Decade to Century-scale. Sun Yat-sen university, Guangzhou, China.

Pritchard, D. W.: Salinity distribution and circulation in the Chesapeake Bay estuarine system.. 1, Mar. Res, 11, 106-123, 1952.

Pritchard, D. W.: The dynamic structure of a coastal plain estuary, J Marine Res, 15, 33-42, 1956.

Ralston, D. K. and Geyer, W. R.: Response to channel deepening of the salinity intrusion, estuarine circulation, and stratification in an urbanized estuary, Journal of Geophysical Research: Oceans, 124, 4784-4802, 2019.

Salles, P., Valle-Levinson, A., Sottolichio, A. and Senechal, N.: Wind - driven modifications to the residual circulation in an ebb - tidal delta: Arcachon Lagoon, Southwestern France, Journal of Geophysical Research Oceans, 120, 728-740, 2015.

Schulz, E., Schuttelaars, H. M., Gr We, U. and Burchard, H.: Impact of the depth-to-width ratio of periodically stratified tidal channels on the estuarine circulation, J. Phys. Oceanogr., 45, 411804097, 2015.

Scully, M. E., Geyer, W. R. and Lerczak, J. A.: The Influence of Lateral Advection on the Residual 
Estuarine Circulation: A Numerical Modeling Study of the Hudson River Estuary, J. Phys. Oceanogr., 39, 107-124, 10.1175/2008JPO3952.1, 2009.

Scully, M. E., Geyer, W. R. and Lerczak, J. A.: The Influence of Lateral Advection on the Residual Estuarine Circulation: A Numerical Modeling Study of the Hudson River Estuary, J. Phys. Oceanogr., 39, 107-124, 10.1175/2008JPO3952.1, 2009.

Scully, M., Friedrichs, C. and Brubaker, J.: Control of estuarine stratification and mixing by windinduced straining of the estuarine density field, Estuaries, 28, 321-326, 10.1007/BF02693915, 2005.

Simpson, J. H., Brown, J., Matthews, J. and Allen, G.: Tidal straining, density currents, and stirring in the control of estuarine stratification, Estuaries, 13, 125-132, 10.2307/1351581, 1990.

Van Maren, D. S., van Kessel, T., Cronin, K. and Sittoni, L.: The impact of channel deepening and dredging on estuarine sediment concentration, Cont. Shelf Res., 95, 1-14, 2015.

Waterhouse, A., Tutak, B., Valle-Levinson, A. and Sheng, Y.: Influence of Two Tropical Storms on the Residual Flow in a Subtropical Tidal Inlet, Estuar. Coast., 36, 1037-1053, 10.1007/s12237-0139606-3, 2013.

Willmott, C. J.: On the validation of models, Phys. Geogr., 2, 184-194, 1981.

Wilson, R. and Filadelfo, R. 1986. Subtidal Current Variability in the Lower Hudson Estuary. Springer Verlag, Berlin, Germany. p. 132-142.

Winterwerp, J. C.: Fine sediment transport by tidal asymmetry in the high-concentrated Ems River: indications for a regime shift in response to channel deepening, Ocean Dynam., 61, 203-215, 2011.

Zhang, R., Chen, L. H., Liu, S. S., Zhang, H. and Lin, G. Y.: Shoreline evolution in an embayed beach adjacent to tidal inlet: The impact of anthropogenic activities, Geomorphology, 346, 106856, 2019.

Zhu, J., Weisberg, R. H., Zheng, L. Y. and Han, S. Z.: Influences of Channel Deepening and Widening on the Tidal and Nontidal Circulations of Tampa Bay, Estuaries \& Coasts, 38, 132-150, 2015. China Normal University, Shanghai, China. 